\title{
Anabases
}

ANABASES Traditions et réceptions de l'Antiquité

6 | 2007

Varia

\section{Une décennie de recherches sur Auguste. Bilan historiographique (1996-2006)}

Frédéric Hurlet

\section{(2) OpenEdition}

1 Journals

Édition électronique

URL : http://journals.openedition.org/anabases/3368

DOI : 10.4000/anabases.3368

ISSN : 2256-9421

Éditeur

E.R.A.S.M.E.

Édition imprimée

Date de publication : 1 octobre 2007

Pagination : 187-218

ISSN : 1774-4296

\section{Référence électronique}

Frédéric Hurlet, « Une décennie de recherches sur Auguste. Bilan historiographique (1996-2006) »,

Anabases [En ligne], 6 | 2007, mis en ligne le 01 janvier 2012, consulté le 20 octobre 2019. URL : http:// journals.openedition.org/anabases/3368 ; DOI : 10.4000/anabases.3368

(c) Anabases 
Anabases 6 (2007), p. 187-218.

\section{Une décennie de recherches sur Auguste. Bilan historiographique $(1996-2006)^{*}$}

FRÉDÉRIC HURLET

IL EST UN FAIT AVÉRÉ que les publications consacrées à Auguste et à la naissance du régime impérial - auquel on donnera le nom de principat - se sont multipliées tout au long de cette dernière décennie. La bibliographie sélective placée en fin d'article en dit long sur l'importance quantitative des études augustéennes, puisqu'elle enregistre près d'une centaine de titres pour la seule période qui va de 1996 à 2006. Outre la nette tendance à l'inflation bibliographique constatée de manière générale ces dernières années, plusieurs autres raisons expliquent un phénomène qui va au-delà d'un simple regain d'intérêt. Si l'époque augustéenne a été considérée dès l'Antiquité comme un moment charnière caractérisé par le passage de la République à l'Empire et Auguste comme un modèle pour les princes à venir ${ }^{1}$, l'analyse de cette période comme un temps de rupture a été confirmée, voire accentuée par la publication dans la seconde moitié du XXe siècle de plusieurs études dont notre génération est tributaire. Dans L'inventaire $d u$ monde, $\mathrm{Cl}$. Nicolet a montré jusqu'à quel point l'avènement du principat augustéen contribua à améliorer les connaissances des Romains dans la représentation de l'oikoumène et la connaissance démographique et économique de leur

* Cet article de synthèse prend en compte les études consacrées à Auguste à partir des réformes de janvier 27 av. J.-C., date à laquelle le surnom Augustus lui fut officiellement conféré. Pour un bilan historiographique relatif essentiellement aux années du triumvirat (43-31 av. J.-C.), on se reportera à Hinard, dans $R H, 1998$, p. 617-642. Mon étude doit un certain nombre de précisions utiles à $\mathrm{A}$. Suspène, notamment pour ce qui touche aux questions de numismatique, et à $\mathrm{B}$. Mineo. Je les remercie très chaleureusement.

1 C'est une analyse qui n'est pas imposée d'emblée et a été développée en particulier par Dion Cassius, cf. SWAN 2004, p. 13-17. 
Empire ${ }^{2}$. Au même moment, P. Zanker a publié un livre magistral qui a mis en relation la documentation archéologique et iconographique d'époque augustéenne avec le programme idéologique du nouveau régime ${ }^{3}$. Cette étude est à mettre en parallèle avec les multiples analyses de $\mathrm{P}$. Gros sur la mise en scène du pouvoir impérial à Rome et dans les cités de l'Empire ${ }^{4}$. G. Alföldy a de son côté souligné dans quelle mesure la mise en place du principat augustéen coïncida avec la naissance d'une culture épigraphique qui se concrétisa notamment par une multiplication du nombre des inscriptions 5 . On doit aux travaux de F. Millar d'avoir montré dans quelle mesure l'État romain est resté, après et en dépit de l'apparition d'un pouvoir monarchique, un État de droit dans lequel le prince prenait d'ordinaire la peine d'associer au processus de prise de décision non seulement le Sénat, mais aussi les comices ${ }^{6}$. Si l'on ajoute l'importance des réformes sociales à la même époque (création de l'ordre sénatorial, redéfinition progressive et lente de la place des chevaliers au service de l'administration impériale ${ }^{7}$ ), on aura compris que la génération actuelle des historiens travaillant sur Auguste a été préparée à poursuivre l'effort accompli et guidée dans cette tâche.

Parmi les raisons qui ont contribué à renouveler notre connaissance du principat augustéen, il faut également prendre en compte la publication de nouveaux documents épigraphiques, numismatiques et papyrologiques. On citera les plus importants d'entre eux : les longues inscriptions provenant d'Andalousie qui retranscrivent tout ou partie de sénatus-consultes et de lois votés en 19 et 20 ap. J.-C. - ce sont les " bronzes espagnols" - et sont connus sous le nom de Tabula Siarensis et de Senatus consultum de Cn. Pisone patre (il est vrai qu'elles datent du principat de Tibère, mais elles fournissent un éclairage sur une idéologie qui reste augustéenne) ${ }^{8}$; un édit d'Auguste daté de 15 av.

2 NiCOLET 1988.

3 ZANKER 1987.

4 GrOs 1996 et 2005, p. 175-187 pour l'époque julio-claudienne.

5 ALFÖLDY 1991. Les dédicaces à Auguste et aux membres de sa famille provenant de Rome ont été publiées en 1996 sous l'autorité d'Alföldy dans la réédition du CIL, VI, 8, 2, Berlin ; cf. aussi le vol. vI, 8, 3, Berlin, 2000 où sont reprises les inscriptions en l'honneur des membres des ordres sénatorial et équestre.

6 Cf. le recueil des principaux articles de Millar sur « la révolution augustéenne " (MillaR 2002).

7 Sur l'ordre équestre sous les Julio-Claudiens, cf. DEMOUGIN 1988 qui précise avec quelle lenteur l'ordre équestre évolua et place la véritable rupture au milieu du Ier siècle ap. J.-C. et à l'époque de la disparition de la première dynastie ; sur l'institution sous Auguste d'un cens sénatorial spécifique (1 million de sesterces), cf. NiCOLET 1976, p. 20-38 ; CHASTAGNOL 1992. Il faut aussi citer les nombreux travaux de R. Syme sur l'aristocratie augustéenne. Pour une édition du sénatus-consulte et de la loi de 19 sur les honneurs funéraires à rendre à la mémoire de Germanicus, cf. CRAWFORD (éd.) 1996 et SÁNCHEZ-Ostiz 1999 ; publié en 2000, un colloque a été consacré en grande partie à ce nouveau document (FRASCHETTI éd. 2000). Pour le SC de Cn. Pisone patre, cf. ECK-Caballos-FernándeZ 1996 (ainsi que STYLOW \& CORZO PÉREZ 1999, p. 23-28 qui présentent une nouvelle copie - la huitième - provenant de Bétique) ; on consultera avec profit la notice de l'AE 1996, nº 885 
J.-C., l'edictum de Pameiobrigensibus, mis au jour dans le Bierzo (Nord de l'Espagne) ${ }^{9}$; un aureus daté de 28 av. J.-C., dont la légende du revers célèbre la restitution " au peuple Romain des lois et des droits " - ou la restitution " des lois et des droits du peuple Romain " - et fait ainsi référence au thème de la restitutio Rei publicae en vigueur dans l'idéologie officielle des années $20^{10}$; l'éloge funèbre d'Agrippa prononcé par Auguste dont un (court) extrait a été conservé sur un papyrus publié pour la première fois en 1970 et qui continue à faire couler beaucoup d'encre ${ }^{11}$; quant au témoignage si fondamental des Res Gestae, il doit faire l'objet très prochainement dans la Collection des Universités de France d'une nouvelle édition confiée à J. Scheid ${ }^{12}$. Devant la profusion bibliographique qui entoure la vie d'Auguste, le moment semble venu de présenter un état de la question qui commente de manière orientée et critique la récente production historiographique sur ce sujet et en propose un classement en fonction des différentes perspectives et des problématiques adoptées.

Il est de multiples manières de rendre intelligible la période de l'histoire politique de Rome qui vit la disparition de la République et l'avènement d'un nouveau régime monarchique placé sous le contrôle d'un Princeps. Relevant depuis l'Antiquité de l'exercice imposé, la biographie représente un genre historiographique à la fois prisé et d'autant plus complexe que la personnalité étudiée a suscité des jugements contrastés. Auguste fait partie de ces figures ambivalentes dont il n'est pas aisé de dresser un portrait fidèle. À ce titre, il a suscité et continue à susciter de nombreux portraits, plus

avec une traduction française de P. Le Roux ; on trouvera également plusieurs traductions anglaises et commentaires de portée générale, fondés sur l'édition allemande du SC de Cn. Pisone patre: M. Griffin, JRS 87, 1997, p. 249-263 ; D. Potter, JRA 11, 1998, p. 437457 ; E. Meyer, $C J 94,1998$, p. 315-324 ; il faut enfin signaler qu'un fascicule spécial a été consacré à ce document dans AJPh $120,1999, \mathrm{n}^{\circ} 1$ (avec une nouvelle traduction de C. Damon et une série d'articles).

COSTABILE \& LiCANDRO 2000, p. 147-235 et AlFÖLDY 2000, p. 177-205. Il faut signaler que l'authenticité de ce document a été mise en doute par LE Roux 2001, p. 331-363 et RICHARDSON 2002, p. 411-415.

Rich \& Williams 1999. La figure du revers représente Octavien en toge, assis sur un siège curule, tenant un rouleau dans sa main droite et un scrinium (un coffret) dans sa main gauche (sur l'iconographie, ZeHNACKER 2003, p. 1-2 défend l'idée selon laquelle le rouleau - uolumen - est le texte de l'édit lu par Octavien lorsqu'il annula en 28 toutes les mesures illégales prises par lui pendant le triumvirat). Le texte de la légende est le suivant : leges et iura p.R. restituit (sur le développement de p.R. - p(opulo) R(omano) ou p(opuli) $R$ (omani) ? -, D. Mantovani penche pour la seconde solution dans une étude à paraittre dans Athenaeum).

11 P. Köln VI, 249.

12 Cette nouvelle édition a été préparée par plusieurs articles portant sur des points précis, cf. SCHEID 2003, p. 359-365 qui propose une nouvelle restitution de la version latine en avançant de bons arguments pour lire au paragraphe 9, 1 [uota pro salute mea], au lieu de la formule traditionnellement acceptée [uota pro ualetudine mea] ; DREW-BEAR \& SCHEID 2005 sur la copie d'Antioche de Pisidie. 
ou moins fondés sur les sources. Le genre biographique a toutefois évolué et à côté d'ouvrages fantaisistes qui se contentent de jugements moraux ou psychologiques éminemment discutables 13 , il y a place désormais pour des enquêtes sérieuses centrées sur la vie d'un individu ${ }^{14}$. Il demeure que s'il est possible de décrire l'État d'Auguste, du moins l'image qu'il voulut lui donner, il est plus difficile dans l'état actuel des sources de percer tous les secrets de ce personnage, et d'évaluer notamment sa sincérité face à l'évolution qui fit d'un chef de faction un prince honoré dans tout l'Empire. Il est significatif que les biographies les plus réussies prennent désormais la peine de dépeindre à plus ou moins grands traits le monde dans lequel Auguste naquit et vécut de manière à rappeler dans quelle mesure les grands hommes étaient eux aussi les produits de leurs temps ${ }^{15}$. Que le savant allemand W. Eck ait consacré une étude à Auguste età son temps (Augustus und seine Zeit) est révélateur de l'attention portée dans les cercles universitaires au contexte sans lequel il n'est pas possible de comprendre le moindre destin individuel 16 .

Il existe d'autres angles d'approche qui ont en commun de définir la nature du principat à travers une étude des pouvoirs, des actions et de l'image d'Auguste tout au long du processus qui vit la république romaine disparaître pour faire place à un nouveau régime qu'Auguste qualifie dans un édit d'optimus status ${ }^{17}$. Il est communément admis que le principat est un régime original qui consacrait une rupture avec l'ancien ordre républicain tout en s'inscrivant dans le prolongement des institutions traditionnelles. Selon que l'on mette l'accent sur les évolutions ou les continuités, l'image d'Auguste en est sortie profondément contrastée. Restaurateur de la République ou monarque absolu 18 , tels sont les deux pôles auxquels ont abouti les recherches historiographiques. La vérité se situe entre ces deux portraits extrêmes, l'inflexion apportée par telle ou telle étude étant essentiellement tributaire des sources prises en considération et de la méthode retenue. Pour une raison historique liée au poids représenté au sein de la tradition universitaire par l'étude des institutions dans l'analyse du système politique, les éléments de continuité l'ont emporté au départ, pendant une grande partie du XXe siècle, lorsqu'il s'est agi de définir la nature du régime mis en place par Auguste. Il faut dire que Th. Mommsen a développé dans son Römisches Staatsrecht une lecture proprement juridique qui a privilégié l'étude des aspects formels des pouvoirs impériaux et influencé diverses générations d'historiens du XXe siècle dont

Par exemple RENUCCI 2003.

Cf. SOUTHERN 1998 et COSME 2005.

Cf. BLEICKEN 1998 ; FrASCHETTI 1998 et KIENAST $1999^{3}$; cf. aussi la courte synthèse de BRANDT 2000, consacrée à Octavien plus qu’à Auguste.

ECK 1998.

Sur cet édit, cf. Suét., Aug., 28, 2 ; sur son contenu et sa datation, cf. GIRARDET 2000b (qui opte pour 29 av. J.-C.).

Pour une analyse d'Auguste comme restaurateur de la République, cf. CASTRITIUS 1982. 
certains passent, volens nolens, pour avoir hérité des méthodes mises au point par le grand historien allemand. Mais on verra que la réaction à l'égard d'une telle approche, tangible dès 1939 dans la Révolution romaine de R. Syme, s'est faite plus vive durant cette dernière décennie. Un des acquis des recherches les plus récentes est d'avoir su intégrer les apports de la sociologie pour montrer dans quelle mesure le pouvoir s'exprimait également à travers la reconnaissance, le loyalisme et l'acceptation que la population exprimait à l'égard d'Auguste. On mesure en outre de nos jours mieux qu'auparavant à quel point le nouveau régime se manifestait de façon matérielle par la diffusion de sa propre image et entretenait avec l'espace des rapports étroits. Ce sont de nouvelles pistes qui ont été ouvertes ces dernières années.

\section{L'ombre de Mommsen : les fondements institutionnels du principat augustéen}

La nature des pouvoirs successifs conférés à Auguste tout au long de sa carrière continue à susciter un débat qui est encore loin d'être clos, mais qui a connu cette dernière décennie des avancées. En matière institutionnelle, la question centrale reste la définition de l' imperium dont le prince ne cessa jamais d'être en possession depuis le 7 janvier 43 av. J.-C. jusqu’à son décès et qui désignait dans son sens technique le plus général la puissance publique la plus élevée, y compris la juridiction et la puissance militaire. Un tel problème a déjà été traité il y a plus d'un siècle dans le Droit public romain de Mommsen, œuvre magistrale qui cherchait à donner une définition légale de l'État romain antique ${ }^{19}$. Cet opus magnum a contribué à placer l'étude des fondements institutionnels du pouvoir impérial à sa naissance au centre des débats historiographiques pendant la plus grande partie du XXe siècle. On pourrait citer sans peine d'innombrables études qui sont revenues de manière lancinante sur l'imperium d'Octavien/Auguste et l'évolution de ses pouvoirs sans parvenir à régler ce problème une fois pour toutes ${ }^{20}$. La légitimité de l'histoire des institutions politiques romaines a été fondée sur le postulat implicite, à mon sens en partie correct, que les questions institutionnelles devraient avoir de l'importance pour nous parce qu'elles en avaient aux yeux des Romains. Une telle justification est longtemps allée de soi et a été pour beaucoup dans la longue postérité des travaux de Mommsen, que des générations d'historiens ont voulu prolonger et

19 Cf. Mommsen $D P R$, tome $\mathrm{V}$ de la traduction française.

20 On citera, exempli gratia, les nombreux travaux de H. Siber, qui prolonge à son corps défendant la méthode mise au point par Mommsen (cf. BEHNE 1999). Plus récemment, la question des pouvoirs d'Auguste a été traitée de façon détaillée par J. Scheid dans JACQUES \& SCHEID 1990, p. 1-46 (ouvrage constamment réédité) et par CROOK 1996 dans la réédition de la Cambridge Ancient History, X. 
affiner ${ }^{21}$. Les conclusions du savant allemand sur la nature du pouvoir impérial ont été très vite critiquées et continuent à l'être aujourd'hui, mais on est loin de s'entendre sur ce qu'il faut au juste lui reprocher. Il y a deux manières foncièrement différentes de procéder à l'analyse critique du Staatsrecht. La première est une critique "interne " qui a consisté et consiste encore à corriger les inexactitudes, intégrer l'apport des sources - notamment épigraphiques - découvertes en nombre non négligeable tout au long du XXe siècle et prendre davantage en compte l'évolution historique des institutions. Plus récente, la seconde est une attaque en règle plus fondamentale qui rejette en bloc à la fois le principe même d'une étude " constitutionnelle » de l'État romain et la démarche qui contribue consciemment ou non à faire du fonctionnement des institutions l'élément central de la vie politique. Il faut partir d'un examen de ces critiques si l'on veut avoir une idée claire de la façon dont sont conçues de nos jours les études sur Auguste et le régime qu'il fonda.

Larticle de fond consacré par J.-L. Ferrary aux pouvoirs d'Auguste représente sans doute l'étude la plus mommsénienne de la fin du XXe siècle et du début du XXIe siècle - étude que n’aurait sans doute pas reniée Mommsen en dépit de maintes divergences sur les résultats obtenus. Nourri par une parfaite connaissance de la bibliographie parue sur ce sujet depuis la fin du XIXe siècle, il s'inscrit dans un débat scientifique pour lequel il présente un état de la question dans des notes infrapaginales très fournies ${ }^{22}$. Les problèmes qui y sont abordés portent tout d'abord sur la définition de l'imperium d'Auguste tel qu'il fut défini au fur et à mesure de réformes successives datées de 27, 23 et 19 av. J.-C. La perspective institutionnelle apparaît d'autant plus nécessaire que le contexte des premières années du principat d'Auguste est celui d'une Res publica restituta soucieuse de ne pas créer de rupture formelle entre les pouvoirs d'Auguste et les antécédents républicains ${ }^{23}$.

La publication récente de l'aureus daté du sixième consulat d'Octavien et célébrant la restitution « au peuple romain des lois et des droits " - ou la restitution " des lois et des droits du peuple Romain "- a permis de reprendre sur nouveaux frais le problème si complexe de l'évolution des pouvoirs d'Auguste en 28 et 27 av. J.-C. Confirmant l'indication chronologique d'un passage des Res Gestae (34.1), la légende de cette monnaie fait ressortir que les mesures de janvier 27 relatives à la question du gouvernement provincial constituaient la dernière étape d'un processus de restitutio Rei

21 Cf. par exemple tout récemment RAINER 2006 dont le manuel reprend le titre choisi par Mommsen : Römisches Staatsrecht. Mais il faut reconnaître qu'en étudiant le droit public pour lui-même et sans envisager ses interactions avec les évolutions de la société romaine, l'ouvrage de Rainer constitue en ce début du XXI e siècle l'exception plus que la règle. FERRARY 2001a.

Sur la définition de la Res publica restituta et les liens entre la restauration de cette nouvelle Res publica et les pouvoirs d'Auguste, cf. l'analyse de FERRARY 2003. 
publicae entamé en $28{ }^{24}$. Pour ce qui est de la teneur des décisions prises en 27 dont la plus connue fut l'attribution à Auguste d'un vaste commandement provincial après que celui-ci eut rendu au Sénat et au peuple romain les provinces qu'il avait détenues en tant que triumvir, l'étude de Ferrary a montré à quel point Auguste veilla à inscrire le gouvernement de l'Empire dans la tradition des commandements extraordinaires de la fin de la République ${ }^{25}$. En la matière, il s'appuya alors sur le précédent pompéien lorsqu'il lui fallut trouver une solution institutionnelle qui lui permit de gouverner à distance un groupe de provinces militarisées. La figure républicaine du grand Pompée fut ainsi érigée au rang de modèle dont le nouveau régime ne s'écarta de façon ponctuelle que par nécessité de s'adapter aux nouvelles conditions de l'époque ${ }^{26}$.

Une autre question, si souvent traitée depuis plus d'un siècle, porte toujours sur le point de savoir de quelle nature était l'imperium du prince tel qu'il fut redéfini en 27. Mommsen avait opté pour un imperium dit proconsulaire, en liaison avec l'existence désormais niée d'une réforme syllanienne qui aurait interdit aux consuls et aux préteurs d'exercer la composante militaire (militiae) de leur imperium 27. Cette thèse a été combattue dès la fin du XIXe siècle par J. Kromayer et H.F. Pelham ${ }^{28}$, mais il est significatif que plus d'un siècle plus tard, ce débat soit loin d'être clos. On compte toujours actuellement des partisans de l'idée mommsénienne selon laquelle l'imperium d'Auguste sur les provinces était proconsulaire en 27 ou l'était devenu à cette date ${ }^{29}$. On dénombre aussi des défenseurs de la thèse inverse, à savoir que l'imperium d'Auguste n'avait pas cessé d'être consulaire ${ }^{30}$. Derrière cette question technique se profile une autre interrogation, plus fondamentale, qui est de savoir si une loi priva le consulat de la composante militaire de ses pouvoirs (en l'occurrence ce qu'on appelle l' imperium militiae) et, si oui, à quelle date. Si plus personne n'attribue aujourd'hui à Sylla une telle mesure comme l'a proposé autrefois Mommsen, des propositions ont été faites récemment pour la dater de l'époque augustéenne. A. Giovannini et $\mathrm{Cl}$. Nicolet, suivis un temps par J.-M. Roddaz, ont proposé plus précisément l'année 27, en relation avec les réformes du mois de janvier qui définissaient les nouveaux pouvoirs d'Auguste 31 . D'autres interprétations ont été par la suite avancées. Girardet a abaissé la date en faisant remonter à l'année 19 ou 18 ce qu'il a appelé l'" Entmachtung des

Cf. Rich \& Williams 1999, p. 169-213 ; cf. aussi RodDAZ 2003a, p. 398-402.

FERRARY 2001a, p. 101-111; cf. aussi FERRARY 2003, p. 419-422.

Sur le modèle pompéien, et ses limites, cf. HuRLET 2006c.

Cf. Mommsen DPR, V, p. 117-118 à propos de l'imperium de 27 av. J.-C. ; cf. aussi Mommsen $D P R$, I, p. 65 et III, p. 107-109 pour la prétendue réforme syllanienne.

Cf. Kromayer 1888, p. 32-34 et Pelham 1888, p. 27-52.

Cf. e. g. BleiCKEN 1990, p. 88-89 et BLEICKEN 1998, p. 326 ; RODDAZ 2003, p. 411-412.

Cf. e. g. GiRARDET 1990, p. 89-126 (auquel s'est partiellement rallié GiOvANNINI 1999, p. 95-102, notamment p. 95) ; FERRARY 2001a, p. 101-113 (avec des différences sensibles par rapport à la position de Girardet).

GIOVANNINI 1983 ; NiCOLET 1992, p. 163-166 ; cf. aussi RodDAZ 1992, p. 204-206. 
Konsulates " ou l'" Entmachtungsgesetz des Konsulates " et lié directement une telle mesure à l'établissement d'un intervalle quinquennal entre le consulat et le proconsulat, daté lui aussi de 19 ou $18^{32}$. Ferrary a soutenu pour sa part qu'une réforme amputant le consulat de l'imperium militiae ne fut jamais votée, pas plus en 23 ou en 19 qu'en 52 ou en 27 av. J.-C. La problématique s'est focalisée à tort, a-t-il ajouté, sur la recherche d'une hypothétique évolution institutionnelle du consulare imperium. Elle doit être recentrée sur le mode de collation des provinces, qui aurait empêché dans la pratique les consuls d'exercer leur imperium militae en fixant un intervalle quinquennal entre l'exercice du consulat et le gouvernement d'une province publique ${ }^{33}$. Il ne faut donc pas imaginer le vote d'une loi qui mit fin de manière légale à un des fondements institutionnels du régime républicain, une telle mesure législative étant en outre incompatible avec le programme de restitutio Rei publicae mis en place à partir de 28. C'est la conclusion la plus vraisemblable dans l'état actuel de nos connaissances 34 .

Innovant davantage que les mesures de 28-27, les réformes de 23 relatives aux pouvoirs d'Auguste (et à ceux d'Agrippa) contribuèrent de façon décisive à la mise en forme définitive du nouveau régime. Que le prince fut alors autorisé à passer le pomerium aussi souvent qu'il le voulait sans être contraint de faire renouveler son imperium à chaque occasion ne fait désormais plus aucun doute 35 . Ce privilège était dans le contexte de l'époque une nouveauté absolue (il ne fut pas conféré à Pompée, qui se trouva pourtant en 54-53 dans une situation proche de celle d'Auguste après son abdication du consulat durant l'été 23) et considérable en ce qu'il permettait à un Romain de cumuler en permanence pouvoir civil et pouvoir militaire ${ }^{36}$. Il doit être analysé comme une des conséquences de l'investiture en 23 de la puissance tribunicienne, qui est devenue le fondement civil des pouvoirs impériaux et sur laquelle je reviendrai. En revanche, aucun consensus n'existe à ce jour sur la supériorité ou non de l'imperium d'Auguste sur les autres détenteurs d'un tel pouvoir (les proconsuls avant tout). Un passage de Dion Cassius a longtemps conduit à penser qu'il avait été investi à cette date d'un imperium maius (ou plutôt d'un maius imperium quam ${ }^{37}$ ), mais la publication de la Laudatio funebris a introduit un élément nouveau en faisant savoir que dans le même contexte, Agrippa, l'alter ego du prince, avait été investi d'un imperium auquel aucun autre n'était supérieur. La nuance est sensible et la question est désormais de savoir si l'imperium d'Auguste tel qu'il fut redéfini à partir de 23 doit être qualifié de maius ("supérieur ») ou d'aequum (" égal »). La teneur de l'éloge funèbre d'Agrippa a conduit

32 GiRARDET 1990, p. 89-126 ; cf. aussi GIRARDET 1992, p. 218-220.

33

34

35

36

37
FERRARY 2001a, p. 101-113.

Cf. dans ce sens RodDaz 2003, p. 412-414 et Hurlet 2006a, p. 28-30.

Pour un état de la question, cf. HURLET 1997, p. 306-308.

L'importance de la réforme de 23 relative au pomerium a été soulignée par FERRARY 2001b. Le caractère relatif - et non absolu - de l'imperium dit maius a été encore rappelé récemment par PASCHOUD 2005, p. 280-282. 
la plupart des spécialistes à privilégier somme toute logiquement la seconde solution 38 , mais il reste à expliquer l'erreur commise à ce sujet par Dion Cassius. Ferrary a présenté une interprétation qui concilie la définition de l'imperium d'Agrippa par Auguste dans la Laudatio funebris avec le témoignage de Dion Cassius sur les mesures de 23 en établissant pour cette année une distinction entre Agrippa et Auguste 39. Le " corégent " aurait été investi en 23 d'un imperium aequum conformément à ce qu'en dit la Laudatio funebris, tandis qu'en accord avec le témoignage de Dion, l'imperium du prince serait devenu supérieur à celui des proconsuls dès cette même année, peu après les mesures relatives à Agrippa ; ce n'est qu'en 18 av. J.-C., au moment de son renouvellement, que l'imperium d'Agrippa serait à son tour devenu maius comme celui d'Auguste. Il est illusoire de trancher définitivement en faveur de l'une ou l'autre des solutions avancées. Quoi qu'il en soit de la réponse à apporter à ce problème spécifique, la question la plus importante est celle de la portée territoriale de l'imperium du prince, que celui-ci soit aequum ou maius. Le passage de Dion Cassius a laissé penser qu'il fut étendu à l'ensemble de l'Empire, laissant à Auguste à partir de 23 la possibilité d'intervenir à sa guise là où il le voulait. Il existe toutefois une autre interprétation, défendue par Ferrary, qui consiste à lier la redéfinition de l'imperium d'Auguste en 23 à sa tournée en Orient et à en restreindre l'application aux provinces publiques qu'il eut à traverser (Sicile, Achaïe, Asie, Pont-Bithynie) et où se posa immanquablement la question de ses rapports avec les proconsuls. Cette solution est, dans l'état actuel de nos connaissances, la plus vraisemblable parce qu'elle inscrit le pouvoir extraordinaire du prince dans le prolongement direct des pratiques républicaines et qu'elle repose sur une série de sources qui subordonnent la supériorité ou l'égalité de l'imperium du prince (ou d'Agrippa, de Germanicus) à sa présence physique dans une province publique.

Autre temps fort des réformes augustéennes, l'année 19 av. J.-C. coïncida avec plusieurs réformes significatives. Le triomphe célébré par L. Cornelius Balbus en mars de cette année était le dernier à avoir été accordé à un général extérieur à la famille impériale. Il a été souligné depuis fort longtemps qu'il s'agissait là d'une rupture dans les pratiques politiques de l'aristocratie romaine, mais il restait à s'interroger sur les fondements institutionnels et les modalités d'une telle évolution. Un lien a pu être établi entre le phénomène de monopolisation impériale du triomphe et la mise en valeur de la suprématie des auspices du prince et des membres de sa famille. L'hypothèse qui a été émise est que le prince fit appliquer avec une plus grande rigueur le droit auspicial, manipulé en la circonstance de manière à affaiblir la légitimité d'auspices pris par des proconsuls qui pouvaient être considérés comme des priuati cum imperio partis dans leur province plusieurs années après avoir exercé le consulat ou la

38 Hurlet 1997, p. 42-52 et 290-294 ; Girardet 2000a, p. 200-219 ; DetTenhofer 2000, p. 110-111 ; PANI 2001, p. 254-256. 
préture ${ }^{40}$. Il n'est pas nécessaire de supposer pour cela le vote en bonne et due forme d'une loi sur ce sujet, le refus par Agrippa de la célébration du triomphe également en 19 a pu servir d'exemplum à imiter censé avoir pour les proconsuls une valeur contraignante ${ }^{41}$. Pour l'année 19, Dion Cassius fait directement référence à une autre mesure en précisant qu'Auguste "prit le pouvoir des consuls à vie de façon qu'il eût toujours et partout les douze faisceaux et qu'il pût s'asseoir sur la chaise curule entre les deux hommes qui étaient consuls à ce moment 42 ". Ce passage a suscité de nombreux commentaires, dont il faut retenir l'interprétation de Ferrary : le prince reçut à cette occasion les insignia du pouvoir consulaire indissociables d'une certaine capacité à exercer l'imperium consulaire dans des domaines déterminés non couverts par la puissance tribunicienne (le cens ou la juridiction) et sans empiéter sur les prérogatives des consuls (par exemple en matière de contrôle des élections consulaires et prétoriennes) ${ }^{43}$.

Le pouvoir civil qu'Auguste exerça à Rome, à l'intérieur du pomerium, ne se limita pas à la composante civile de l'imperium consulaire - l'imperium domi - qui lui fut conféré sans interruption en tant que consul de 31 à 23, puis à partir de 19. Il faut compter avec une autre composante civile des pouvoirs impériaux, la puissance tribunicienne, qui était le pouvoir détenu par les tribuns de la plèbe. D'après l'opinio communis, qui repose principalement sur le témoignage de Dion Cassius, il en fut investi durant l'été 23 , en compensation de l'abdication du consulat, à titre viager moyennant un renouvellement annuel purement formel après s'être fait attribuer précédemment diverses compétences tribuniciennes (la sacrosainteté et le ius subsellii en 36, le ius auxilii en 30). On ne peut pas écarter définitivement l'idée, fondée sur le témoignage d'Appien, selon laquelle Octavien reçut la puissance tribunicienne à vie dès 36 . Dans cette perspective, la mesure de 23 aurait introduit uniquement la pratique du renouvellement annuel de ce pouvoir et doit être interprétée comme un expédient destiné à combiner le principe de la perpétuité avec celui de l'annalité pour mettre fin aux difficultés institutionnelles posées par l'itération répétée du consulat ${ }^{44}$. Il faut toutefois rappeler que, comme le souligne Dion Cassius (53.33.2), l'investiture de la puissance tribunicienne se justifie autant - sinon plus - par la crainte de voir l'aristocratie sénatoriale réagir à une monopolisation impériale du consulat qui la privait

40 Hurlet 2001 et Hurlet 2006a, p. 161-177. Sur les auspices d'Auguste et des proconsuls, cf. aussi TARPIN 2003 qui traite de la question du refus des dépouilles opimes à M. Licinius Crassus et où l'on trouvera notamment p. 287-289 une excellente analyse d'un passage du De diuinatione de Cicéron (2.76). 
concrètement du gouvernement des provinces consulaires que par les difficultés institutionnelles créées par l'exercice continu de la magistrature suprême qu'il n'était pas d'usage de détenir aussi longtemps. Les raisons du choix de ce pouvoir civil ont été également discutées. La puissance tribunicienne a été analysée comme le vecteur d'une politique " populaire 45, » Octavien/Auguste s'inscrivant ainsi dans la continuité des tribuns séditieux de la fin de la République et de son père adoptif Jules César, mais cette explication n'est ni la seule, ni la plus convaincante. Elle était également un instrument institutionnel qui donna à Auguste les moyens de contrôler le Sénat et l'assemblée du peuple à partir du moment où il renonça à être consul ${ }^{46}$. Elle légitimait enfin sa présence à l'intérieur des limites du pomerium, l'exercice des prérogatives tribuniciennes ne se concevant pas extra Vrbem. Ce pouvoir civil ne s'imposa pas d'emblée comme l'élément central des pouvoirs impériaux, le summi fastigii uocabulum d'après les termes de Tacite (Ann., 3.56.2). Il ne fut considéré comme l'indication officielle de la position impériale que progressivement ${ }^{47}$.

Les pouvoirs impériaux ne pouvaient se concevoir sans un pôle religieux indissociable dans l'Antiquité de toute forme de vie publique. On sait qu'Auguste finit par devenir prêtre des quatre grands collèges religieux de Rome - sans compter les autres sacerdoces et sodalités auxquels il appartenait. Cette pratique, si elle n'était pas en soi illégale, était toutefois nouvelle, le pouvoir sacerdotal étant auparavant morcelé. Le cumul des fonctions militaires, civiles et religieuses ne doit plus étonner depuis que les travaux de J. Scheid ont montré que les aspects politiques et sociaux prédominaient dans l'expression de la religion romaine, qu'il faut définir comme une orthopraxie reposant notamment sur une série de rites célébrés dans le cadre de la cité ${ }^{48}$. La dernière décennie a vu la publication de plusieurs travaux qui viennent affiner cette analyse. Les fragments épigraphiques qui retranscrivent le texte important du protocole augustéen des jeux séculaires de 17 av. J.-C. (présidés par Auguste et Agrippa en tant que XVvirs) ont fait tout d'abord l'objet d'une nouvelle édition commentée ${ }^{49}$. Nous disposons en outre désormais d'un excellent outil de travail sous la forme de fastes sacerdotaux qui

45 Cf., dans ce sens, DETTENHOFER 2000.

46 FERRARY 2003, p. 426-427.

47 Sur cette question, cf. LACEY 1996, p. 154-168 [réédition d'un article de 1979 publié dans les $J R S$ ] qui précise que la puissance tribunicienne ne fut pas utilisée comme formule de datation avant l'année 18 av. J.-C., mais cette analyse doit être nuancée sur ce point précis : la puissance tribunicienne est (probablement) mise en avant par des monnaies de bronze frappées à Rome et à coup sûr par des monnaies du légat d'Espagne T. Carisius entre 23 et 20, une date très haute (RIC, Aug., 11a-25 et 394).

Sur cette définition, à laquelle était parvenu Scheid depuis longtemps - au moins dès la première édition de son ouvrage sur Religion et piété publié en 1985 et réédité en 2001 -, cf. de nouveau récemment SCHEID 2005 (cf. notamment p. 189-209 où il revient sur les parentalia d'après les honneurs accordés à Caius et Lucius César). SCHNEGG-KÖHLER 2002. 
enregistrent tous les prêtres de Rome et leur personnel pour la période qui va de $300 \mathrm{av}$. J.-C. à 499 ap. J.-C. 50 . Les travaux de J. Rüpke ont également souligné à quel point et de quelle manière le calendrier fut instrumentalisé notamment par l'insertion de fêtes liées au nouveau régime et une plus grande diffusion des fastes, contribuant à ce que l'on peut qualifier d'" augustification"du temps 51 . Enfin, il faut signaler la parution d'une étude ponctuelle de J. Scheid qui tire toutes les implications de l'élection somme toute tardive de l'élection d'Auguste comme grand pontife (le 6 mars 12 av. J.-C.) ${ }^{52}$. Il y est montré dans quelle mesure et comment le prince contourna en matière de procédure les situations et les décisions qui devaient comporter une consultation et un avis en bonne et due forme du collège pontifical tant que celui-ci resta placé sous la présidence de Lépide, soit durant les premières années du nouveau régime. Ce contexte si particulier explique pourquoi il fallut attendre la mort de ce dernier en 13 av. J.-C. pour que soient adoptées une série de mesures par lesquelles Auguste clôtura le programme de restauration de la religion et s'appropria dans le même temps davantage le temps et l'espace de Rome. Ce n'est en effet pas un hasard si datent des mois ou des années qui suivirent l'élection au grand pontificat les événements importants suivants : restauration du flaminat de Jupiter (12 av. J.-C.) ; transformation d'une partie de sa demeure du Palatin en un espace public abritant un autel ou un petit temple de Vesta (12 av. J.-C.) ; sans doute transfert des livres sibyllins au temple d'Apollon du Palatin (12 av. J.-C. ou peu après) ; réforme du calendrier qui changea le nom du mois de Sextilis (sixième) en Augustus en souvenir de victoires remportées en août par le prince (8 av. J.-C.).

Les pouvoirs des membres de la famille d'Auguste qualifiés d'ordinaire de " corégents " (Agrippa, Drusus l'Ancien, Tibère, Caius César, Germanicus et Drusus le Jeune) ont été également étudiés de manière détaillée. A été consacrée à ce sujet par mes soins une thèse de doctorat dont les deux premières parties ont traité des questions proprement institutionnelles 53 . Il ressort que l'investiture de l'imperium par le biais d'un sénatus-consulte ratifié par une loi fit, dans un premier temps, de ces proches du prince des chargés de missions extraordinaires (Agrippa de 23 à 18, Tibère de 11 à 6 , Caius César de 1 av. J.-C. à 4 ap. J.-C., Germanicus de 11 à 19 et Drusus le Jeune de 17 à 22). Seul l'octroi, dans un second temps, de la puissance tribunicienne leur donna le statut de collègue du prince, bien attesté par des documents tels que les fastes « impériaux " de Brescia et de Luni (Agrippa de 18 à 13, Tibère de 6 à 1 av. J.-C. et de 4 à 14 ap. J.-C., sous Tibère Drusus le Jeune de 22 à 23). La troisième partie de mon livre a cherché toutefois à montrer qu'il fallait dépasser l'analyse institutionnelle pour comprendre la position des collègues du prince sous tous ses aspects.

50 RÜPKE 2005. La parution de ce livre laisse espérer la réalisation de son pendant politique sous la forme d'un ouvrage qui prolongerait, pour l'époque augustéenne, les listes des magistrats établies pour l'époque républicaine par Broughton.

52 SCHEID 1999.

53 HuRLET 1997. 
Les pouvoirs d'Auguste et de ses proches continuent à intéresser. La question de leur définition et de leur évolution est désormais au centre d'un débat dont on vient de voir qu'il a donné lieu à différentes opinions contradictoires. Quelles que soient leurs divergences, tous les travaux qui viennent d'être cités apparaissent comme un prolongement d'un courant de la recherche qui place au premier plan l'étude des institutions, dans la lignée de Mommsen. Mais il est une autre manière, différente, de rendre compte de la prise du pouvoir par Auguste et de la nature du nouveau régime. Il faut pour cela étudier la naissance du principat à partir d'une perspective qui ne soit plus exclusivement institutionnelle.

\section{La réaction à Mommsen : les travaux sur le consensus}

L'historien anglais R. Syme, l'auteur de La Révolution romaine (1re éd., 1939), constitue l'exemple le plus représentatif d'un savant qui sut assez vite prendre ses distances par rapport à l'héritage mommsénien en se montrant plus sensible aux réalités pragmatiques du pouvoir quà toutes les complexités de sa mise en forme. Dans le prolongement des travaux de Fr. Münzer, il a fait une utilisation intensive de la prosopographie, méthode féconde qui se fonde sur la mise en série des noms de personnages de l'Antiquité. Il a montré que le changement le plus profond était à chercher dans la transformation sous Auguste de la classe dirigeante de Rome avec la disparition progressive de l'ancienne noblesse proprement romaine attachée au régime oligarchique et la promotion d'une nouvelle noblesse élargie à l'ensemble de l'Italie (cf. aussi The Augustan Aristocracy, 1986). Il serait toutefois vain d'opposer à toute force Syme à Mommsen. Il vaut mieux retenir à ce sujet que sans être nécessairement contradictoires ou exclusives, ces deux tendances attestent que l'analyse du système politique en vigueur à Rome, sous la République ou sous l'Empire, peut être conçue dans deux perspectives différentes selon qu'on se fonde sur la légitimité du pouvoir ou sur sa pratique ${ }^{54}$.

Des reproches plus fondamentaux ont été émis ces dernières années à propos de la méthode de Mommsen et de tout l'héritage mommsénien. Elles ont pour conséquence de ne plus parler de l'imperium (ou de façon très limitée) quand il est question du pouvoir à Rome et de s'intéresser à des réalités extra-institutionnelles tels que le phénomène du consensus, la naissance et le développement d'une cour, les formes d'expression et de répression de l'opposition au pouvoir impérial, le mode de communication politique orale et écrite 55 . Il s'agit là de réactions qui conduisent à renouveler nos problématiques et qui sont pour tout dire salutaires, mais dont on verra qu'elles

54 Comme l'a fait remarquer LINDERSKI 1990, p. 51-52.

55 Sur le mode de communication politique orale et écrite à l'époque augustéenne, cf. Cresci Marrone (éd.) 1998. Sur la naissance d'une cour impériale, cf. WallaceHadrill 1996 et WinTERLING 1999 ; cf. aussi Hurlet 2000 pour un état de la question. Les autres thèmes seront développés infra. 
sont également excessives dans un certain nombre de cas. Le paradoxe est que c'est principalement d'Allemagne que sont venues les critiques les plus dures et les plus fondamentales à l'égard de Mommsen et de son manuel de Droit public. Leur dénominateur commun est de rejeter les anciens concepts de légalité, légalisation, légitimité, légitimation, constitution, révolution, souveraineté populaire, qui ont été forgés par la science historique allemande du XIXe siècle et dont il est légitime de se demander s'ils peuvent être effectivement appliqués à l'histoire ancienne.

E. Flaig est celui qui a formulé les critiques les plus dures à l'égard de l'œuvre de Mommsen et de tout un courant de la recherche historique à laquelle il donne le qualificatif de staatsrechtlich orientierte Forschung. Partisan d'une interprétation sociologique du pouvoir impérial, il est parti d'une étude consacrée au phénomène de l'usurpation sous le Haut-Empire pour proposer une analyse globale qui accorde au consensus une place centrale dans la définition du régime impérial ${ }^{56}$. Le raisonnement de Flaig part d'un constat simple. En définissant l'usurpation comme un défi lancé à l'empereur en fonction par un prétendant au pouvoir suprême et en la présentant comme une réaction politique naturelle, il fait apparaitre le principat comme un régime qui aurait reposé non pas sur ce que nous appelons la légitimité de l'institution impériale, mais sur l'adhésion des acteurs principaux de la vie politique à la personne même de l'empereur. L'existence et la nature de ce type de crise politique laisseraient entrevoir l'essence du pouvoir impérial tel qu'il fut fondé par Auguste et le conduisent à définir le principat comme un "Akzeptanz-System ». Ce système politique trouve sa formulation antique dans la notion de consensus universorum, expression bien attestée par les sources qui renvoie à l'adhésion des principales forces en présence dans la société romaine - forces que Flaig appelle « les secteurs déterminants du système politique " : en l'occurrence l'armée, l'ordre sénatorial et la plèbe urbaine, qui pouvaient marquer de manière formelle leur ralliement au prétendant respectivement par l'acclamation, le sénatus-consulte et la loi comitiale. Définir la nature du régime impérial sous un tel angle revient à donner à l'usurpation une dimension tout à fait particulière et originale. Elle n’est désormais plus un élément dépourvu de quelque règle que ce soit, ni un phénomène politique dans lequel la structure révolutionnaire chère à Mommsen apparait au grand jour. Elle devient le moyen politique le plus naturel par lequel un prétendant essaie de détourner à son profit le consensus de tous les secteurs de la vie politique.

Le mode d'analyse développé par Flaig a pour résultat principal de minorer toute approche de type juridique, voire de la condamner et de l'écarter au motif qu'elle ne permet pas de définir l'essence d'un régime politique, que ce soit la république romaine ou le principat augustéen. C'est ce qu'il affirme de façon brutale lorsqu'il écrit que «le prétendu droit public est sans importance 57 ». Ce parti pris est la conséquence ultime 
de l'importance accordée au phénomène de l'unanimité. Le consensus renvoie en effet d'emblée à un autre domaine que celui du droit en déplaçant l'analyse historique sur le terrain des rituels politiques qui scandaient la vie sociale des Romains. Cette nouvelle perspective le conduit à définir le droit public comme un " code " dont l'étude n'apporterait rien de fondamental à la compréhension des phénomènes proprement politiques. On mesure mieux ainsi le fossé qui sépare de telles conceptions des travaux d'un grand nombre de ses devanciers. Alors que l'histoire de la République et de l'Empire incluait d'ordinaire une dimension institutionnelle qui cherchait toujours à décrire les formes prises par le pouvoir à une époque ou une autre, Flaig s'épargne cette peine pour concentrer directement son attention sur la pratique politique, seule manière selon lui de définir la nature et l'essence même du pouvoir à Rome. Les travaux de Flaig, pour provocateurs qu'ils soient, n'ont pas laissé et ne laissent pas indifférents 58 . Ils ont trouvé en France un défenseur en la personne de P. Veyne, qui a défini l'empereur " comme un mandataire de la collectivité, chargé par elle de diriger la République " et placé dans une situation inconfortable dans la mesure où le contrat le liant aux principales forces politiques pouvait être à tout moment rompu 59 . Pour le monde anglosaxon, il faut signaler que l'analyse par Gr. Rowe des nouveaux sénatus-consultes tibériens (Tabula Siarensis et SC de Cn. Pisone patre) va dans le même sens. L'originalité de ce livre est de ne pas se limiter, comme l'a fait Flaig, à l'étude des seuls rapports entretenus par le prince avec le Sénat, la plèbe urbaine et l'armée. Rowe élargit le cercle de la vie politique aux chevaliers et aux cités de l'Empire, acteurs à part entière par lesquels Auguste voulut être reconnu et accepté ${ }^{60}$.

Quelles que soient les nuances à apporter aux travaux de Flaig, le principal acquis de ses travaux est d'avoir placé la recherche du consensus au centre de toute analyse du régime politique en vigueur à Rome. S'il est loin d'être le premier à avoir souligné l'importance du consensus uniuersorum 61 , il est toutefois sans conteste celui qui a su en tirer

58 Sur l'importance de l'ouvrage de E. Flaig sur l'usurpation sous le Haut-Empire, mais aussi son caractère iconoclaste, cf. la présentation qu'en donne SPÄTH 1999, p. 1137-1140 où il est précisé que "Flaig prend ses distances avec la tradition de l'histoire du droit constitutionnel qui, à la suite de Mommsen et de Kromayer, proposa diverses constructions d'une imaginaire "constitution romaine" "(p. 1140) ; il ajoute que "loin d'être un problème juridique ou de légitimité, le principat se forme dans les pratiques de la communication politique ».

VEYNE 2002a et VEYNE 2005, p. 15-78.

60 Rowe 2002.

61 Il est vrai que dès 1937, A. von Premerstein a déjà consacré dans son ouvrage sur l'essence même du principat un long chapitre aux fondements sociologiques du principat (Vom Werden und Wesen des Prinzipats, Munich) ; point important pour notre propos, il a fait des serments à l'empereur la source principale d'une autorité qui aurait recherché l'adhésion la plus large et instauré, par ce biais, avec les gouvernés, les mêmes relations qu'entre un patron et ses clients. Cf. aussi INSTINSKY 1940 et LE GALL 1985. 
toutes les implications dans la définition du régime impérial dans des termes qui relèvent de la science politique. Un des reproches que l'on peut lui adresser est de ne pas s'être livré à un examen précis des attestations du terme consensus (uniuersorum) sous Auguste. L'insistance avec laquelle Auguste fait valoir dans les Res gestae que sa position à la tête de l'État était justifiée par l'unanimité à son égard est connue depuis longtemps. S'y ajoute désormais le témoignage de l'éloge funèbre d'Agrippa prononcé par Auguste en 12 av. J.-C., dans lequel il est précisé que le fidèle second et gendre du prince avait été élevé au rang suprême non seulement par le zèle d'Auguste et ses propres mérites, mais aussi " par le consentement de tous les hommes 62 ». La légende du revers d'un denier va dans le même sens en représentant une colonne sur laquelle était gravée l'inscription suivante : Imp(eratori) Caes(ari) Aug(usto) comm(uni) cons(ensu) 63. Le consensus uniuersorum constitue également une partie intégrante du message délivré par le Sénat romain dans les bronzes d'époque tibérienne découverts en Bétique (la Tabula Siarensis et le Senatus consultum de Cn. Pisone patre). Si les nouveaux documents confirment l'analyse de Flaig plus qu'ils ne l'infirment, la question est de savoir comment l'historien peut prendre en compte un tel concept. Accepter l'idée, incontestable, que le consensus était un élément constitutif du principat augustéen et occupait une place centrale dans l'idéologie impériale ne revient pas à valider toutes les conclusions auxquelles Flaig est parvenu. La centralité accordée à l'unanimité, loin de résoudre tous les problèmes, pose deux nouvelles questions majeures : l'historien doit-il se désintéresser de l'étude des institutions romaines et abandonner cette matière aux seuls juristes sous le prétexte que le droit n'a pas vocation à expliquer les phénomènes proprement politiques ? Comment articuler la recherche par Auguste du consensus avec les autres fondements idéologiques du nouveau régime comme la victoire - sur laquelle repose une unanimité de circonstance - ou de réalités telles que l'existence d'une opposition ? Les lignes qui suivent ont pour objet d'ouvrir un débat dont on peut espérer qu'il soit prolongé.

Le désintérêt de Flaig à l'égard de toute démarche juridique est excessif. Il n’a pas jugé bon de répondre à la question de la place à accorder à l'étude des institutions en parfaite logique avec une démarche qui fait table rase de tout l'héritage mommsénien. Ce parti pris est d'autant plus regrettable qu'à la différence de la plupart de ceux qui s'étaient intéressés avant lui au consensus (notamment L. Wickert, E. Kornemann ou H.U. Instinsky), il a formulé une remarque intéressante, et correcte à mon sens, en précisant qu'un tel phénomène s'exprimait par des manifestations formelles : «Was existiert, hat Form 64 ». Il a établi une liste de rituels politiques qui mettaient en avant

62 P Köln VI, n 249.

63 RIC, Aug., 358. Il est à noter qu'on a voulu lire sur cette monnaie la formule comm(uni) cons(eruatori), mais la référence au comm(unis) cons(ensus) me paraît préférable. Cf. aussi RIC, Aug., 418 dont le revers représente un cippe sur lequel est gravée la formule étrange c.c. Augusti (par référence au communis consensus ?).

FLAIG 1992, p. 198. 
l'unanimité autour du prince : l'acclamatio, la salutatio publique, les cérémonies de la profectio ou de l'aduentus, les ludi, les réunions des comices et du Sénat ... Ce sont précisément les formes prises par de telles manifestations du consensus que l'historien des institutions politiques peut à bon droit étudier. Flaig refuse d'entrer dans de telles considérations, mais cette attitude a pour effet de laisser dans l'ombre une partie signifiante de la réalité historique. C'est ainsi que la manière dont le consensus se manifeste est non seulement intéressante à analyser en soi, elle fournit en outre de précieuses indications sur les modalités de la communication politique et les rapports de force existant à tel moment. Il est, en effet, avéré que le consensus uniuersorum ne revêt pas une forme unique. Il s'exprime de différentes façons en fonction du contexte politique. L'association du sénatus-consulte et d'une loi comitiale représente la forme légale la plus souvent attestée de l'accord général qui pouvait se nouer entre les sénateurs et le peuple Romain. Elle ne constitue pas le seul mode possible d'expression du consensus ${ }^{65}$. On rappellera par exemple qu'en 2 av. J.-C., Auguste fut appelé " père de la patrie " par le Sénat, l'ordre équestre et le peuple Romain tout entier à la suite d'acclamations successives par chacun de ces corps constitués ${ }^{66}$; le Senatus consultum de Cn. Pisone patre élargit le cercle de l'unanimité en soulignant que le Sénat, l'ordre équestre, la plèbe et les soldats s'étaient unis en tant que groupes influents de la société romaine pour condamner Pison et exprimer leur piété à l'égard de la mémoire de Germanicus et de la famille impériale ${ }^{67}$; s'y ajoute le serment prêté à l'empereur et à sa famille, qui apparaît comme l'extension maximale du consensus à l'égard du régime impérial dans le sens où les provinciaux marquaient eux-mêmes leur attachement au pouvoir en place à Rome ${ }^{68}$. On voit donc que les modalités et les acteurs de ce consensus uniuersorum variaient en fonction du contexte. Pour ce qui est des acteurs, il faut compter non seulement l'armée, le Sénat, le peuple Romain, mais aussi l'ordre équestre et les provinciaux. Le consensus uniuersorum n'a pas fini de faire parler de lui, à juste titre d'ailleurs, à condition de dépasser le constat selon lequel il s'agit d'un des fondements idéologiques du principat. Il faut se demander également qui se rangeait unanimement derrière le pouvoir en place à Rome ${ }^{69}$ et de quelle manière un tel rituel politique était célébré.

65 Sur la question des modalités du consensus uniuersorum, cf. déjà ADCOCK 1951, p. 134.

66 Res Gestae, 35.

67 Cet aspect du message politique délivré par le Senatus consultum de Cn. Pisone patre a été bien mis en évidence par ECK-CABALLOS-FERNÁNDEZ 1996, p. 298-303.

Pour une analyse qui fait du serment à l'empereur une extension au-delà de Rome de l'idéal de consensus uniuersorum, cf. HURLET 2002, p. 171-172.

69 Dans le cadre de l'importance accordée au consensus, Flaig s'est limité à étudier les interactions entre ce qu'il appelle les " trois secteurs déterminants du système politique ", à savoir l'armée, le Sénat et le peuple Romain. Il faudra élargir cette analyse et s'interroger, par exemple, sur le rôle de l'ordre équestre et des provinciaux dans l'élaboration de ce consensus. 
Nul doute que les historiens des institutions politiques pourront (et devront) être sollicités pour répondre à cette dernière question.

\section{Du consensus à la dissensio}

Pour cardinal qu'il soit, le concept de consensus ne peut rendre compte seul de la nature du régime augustéen. Il a été utilisé si (trop) largement qu'il a été considéré comme envahissant ${ }^{70}$, voire étouffant. À ce titre, il a été et est toujours critiqué ${ }^{71}$. Le problème majeur est que le consensus uniuersorum est par définition universel et s'il y eut des voix dissidentes, nous ne les entendons pas ou peu ${ }^{72}$. Il s'agit donc là d'un idéal vers lequel l'État romain a de tout temps tendu, mais qu'il n'a jamais atteint complètement. L'unanimité parfaite est un absolu impossible à réaliser. Cette remarque vaut en particulier pour Auguste. Il y avait un équilibre à rétablir, ce qui fut fait avec la parution de trois ouvrages consacrés par M.H. Dettenhofer, Fr. Rohr Vio et I. Cogitore au phénomène de l'opposition au pouvoir impérial et de la conspiration contre le prince ${ }^{73}$. Du consensus on est passé à l'étude de la dissensio et de ses principales manifestations. Le point commun de ces trois monographies est d'étudier les résistances suscitées par l'ascension politique d'Octavien/Auguste non pas pour elles mêmes, mais comme un moyen de dessiner en creux les modes d'expression d'un nouveau pouvoir amené à étouffer toute forme de contestation pour pouvoir survivre. Pour le reste, elles ont privilégié chacune une perspective spécifique.

L'ouvrage de Dettenhofer a adopté un angle de vue original, mais aussi discutable, qui cherche à suivre étape après étape les moyens dont Auguste a su contourner les nombreux obstacles successifs placés sur son chemin pour imposer son autorité et instaurer une dynastie garante de la continuité du nouveau régime. Rejetant le terme d'" opposition " pour ses résonances contemporaines et parce que ce terme ne peut s'appliquer qu'à l'encontre d'un système politique déjà bien établi, elle privilégie les concepts de " résistance " et de " concurrence " pour analyser les différents conflits, voire les complots, qui cherchaient à affaiblir la position du prince et qui pouvaient également viser les membres en vue de la dynastie. Si cet ouvrage est loin d'être le premier consacré à un tel sujet (cf. la monographie de Sattler ou l'article de RaaflaubSamons, dans Between Republic and Empire, éd. par K.A. Raaflaub et M. Toher,

70 Sans que l'on puisse y reconnaître de lien direct avec les travaux de Flaig, le titre des catalogues de l'exposition organisée à Rome en 1995, I luoghi del consenso imperiale. Il foro di Augusto. Il foro di Traiano, montre à quel point le concept de consensus a été en vogue. Cf. par exemple, pour une critique récente du consensus, Gros 2001, p. 140.

Sur l'expression du consensus à travers les monuments de Rome d'époque augustéenne, mais aussi sur les limites et la complexité d'une telle notion, cf. les remarques récentes de T. Hölscher et de F. Millar dans GiovanNini (éd.) 2000, p. 247-259, 275-277 et 328. DetTenhofer 2000 ; RoHr Vio 2000 et Cogitore 2002. 
Berkeley, 1990, p. 417-454), il se distingue malgré tout en ce qu'il retrace l'évolution du principat augustéen à l'aune des résistances suscitées à Rome par la prise du pouvoir d'Octavien/Auguste et toutes étouffées. Autre particularité, Dettenhofer ne retient pas les années 19/17 comme le terme des conflits avec l'aristocratie sénatoriale et le point de départ du consensus uniuersorum. Elle considère au contraire qu'un phénomène de concurrence a perduré après la cérémonie des Jeux séculaires, mais il s'est déplacé peu à peu du Sénat vers le cœur de la dynastie, signe d'une confusion progressive entre la Res publica et la domus Augusta. Elle refuse donc de suivre l'interprétation traditionnelle qui voit dans les mesures de janvier 27 un " compromis » avec le Sénat et préfere retenir l'idée d'une sourde contestation à l'encontre d'un Prince qui aurait imposé ses pouvoirs. Par la suite, si la résistance de nombreux sénateurs de l'ancienne noblesse s'était faite de manière plus feutrée et de moins en moins ouverte à partir des années 10 av. J.-C., elle ne se serait pas pour autant éteinte. Le nouveau régime n'aurait été en fin de compte définitivement accepté par l'aristocratie romaine que dans le courant des années 8 à 14 ap. J.-C. Élève de Chr. Meier, Dettenhofer repousse au bout du compte la solution trouvée par Auguste à la " crise sans alternative » jusqu’aux dernières années $\mathrm{du}$ Principat et rejette l'interprétation de tous ceux qui datent la consolidation du nouveau régime des années 10 av. J.-C. (cf. notamment W.K. Lacey, pour lequel le consensus uniuersorum fut réalisé en $19{ }^{74}$ ). Souligner les difficultés rencontrées par le Princeps non seulement au moment de sa prise du pouvoir, mais aussi tout au long du " siècle d'Auguste " est une entreprise légitime qui contribue à restituer au Principat augustéen sa dimension évolutive, mais le risque est grand d'assimiler des conflits ponctuels à une résistance générale à l'encontre du nouveau régime. Si l'ouvrage de Dettenhofer est bien informé, il n’a pas su totalement éviter cet écueil et pèche par un esprit de système qui ne résiste pas toujours à un examen attentif des sources.

Louvrage de Rohr Vio recense les différentes formes de contestation du pouvoir d'Auguste en montrant dans quelle mesure la mémoire du dissensus fut instrumentalisée et manipulée pendant les événements et après coup. La monographie de Cogitore s'inscrit dans le même courant d'idées, avec cette double spécificité qu'elle prolonge de telles recherches jusqu'à la fin de l'époque julio-claudienne et se concentre sur une forme particulière d'opposition, la conspiration. L'ouvrage s'articule autour de quatre chapitres. Le premier est consacré au " matériau littéraire " et définit la méthode à suivre dans le traitement de la documentation. Il s'agit de reconstituer le schéma choisi par les textes littéraires, qui constituent la majorité écrasante des sources, pour présenter une conspiration à travers ce qui s'apparente plus ou moins étroitement à un récittype. Y sont analysés les éléments récurrents tels que le canevas adopté pour décrire cette forme d'opposition au pouvoir, les passages obligés, les lieux communs ou encore la terminologie. Les conspirations connues pour la période qui va de 43 av. J.-C. à la 
mort de Néron, au nombre d'une trentaine dans l'état de notre documentation, sont ensuite passées en revue d'une manière qui se veut à la fois événementielle et analytique. Elles sont regroupées en trois catégories qui forment respectivement le thème des trois chapitres restants. Une première série cohérente et bien attestée pour l'époque augustéenne rassemble tous les projets qui justifiaient l'élimination du prince par le désir, sincère ou non peu importe, de rétablir la République. Cogitore y souligne les ambiguïtés des références à la République et aux valeurs qui lui sont attachées lorsqu'Octavien était en butte à des complots dans un contexte de concurrence avec Antoine. Le chapitre suivant s'intéresse aux conspirations qui semblent avoir été montées de toutes pièces pour mettre en valeur le prince et ses qualités (son souci du bien public, la clémence d'Auguste dans le cas de Cinna) ; il démonte en particulier les mécanismes qui ont conduit le pouvoir ou les sources postérieures aux événements à inventer une opposition qui n'existait pas ou qui n'était pas du moins aussi dangereuse qu'on a pu le prétendre. Le dernier chapitre analyse les conspirations dynastiques. L'influence exercée directement ou non dans l'apparition des complots par les princesses, notamment les deux Julies, est mise en évidence, ainsi que les manœuvres du pouvoir impérial pour écarter de la dynastie des membres devenus indésirables et faire disparaître les successeurs potentiels (cf. Agrippa Postumus). L'existence de dissensions au sein de la famille impériale est une réalité qui est à mettre en parallèle avec les avatars de l'histoire de la dynastie fondée par Auguste et que d'autres travaux de cette dernière décennie ont contribué à éclairer pour de multiples aspects ${ }^{75}$. Sur le fond, l'ouvrage de Cogitore livre une analyse en profondeur dont l'idée de fond a priori paradoxale est que cette forme d'opposition renforçait la légitimité du pouvoir plus qu'elle ne l'affaiblissait en lui donnant l'occasion de s'affirmer. Une telle conclusion est le résultat d'une méthode qui consiste à mettre en série toutes les conspirations d'époque julio-claudienne connues par les sources et à les étudier non pas pour elles-mêmes, mais en fonction de ce qu'elles nous apprennent sur la nature du pouvoir impérial et sa légitimité. Dans ces conditions, la question de la réalité de telle ou telle conspiration, souvent posée avec raison par les historiens contemporains qui soupçonnent le pouvoir impérial d'avoir inventé dans son intérêt des tentatives d'assassinat du prince ou recomposé a posteriori le récit des événements, passe au second plan. Le problème central abordé dans ce livre est celui de l'image du pouvoir impérial telle qu'elle se dégage des raisons de porter atteinte à la vie du prince, du déroulement des faits et du comportement du pouvoir impérial à ces occasions. Du phénomène de la dissensio, on passe à l'étude de la manière dont le pouvoir impérial aimait à se représenter ou se faisait représenter,

75 Sur les relations conflictuelles entre Tibère et les princes de la jeunesse, cf. HURLET 1997 ; sur la guerre sourde et larvée qui opposa à partir de 4 ap. J.-C. les deux branches de la domus d'Auguste - claudienne autour de Tibère, julienne autour d'Agrippa Postumus -, notamment autour de la dédicace du temple de Castor et Pollux, cf. SUSPÈnE 2001. 
étant entendu que pour l'histoire antique, nous sommes finalement moins informés sur la réalité des événements que sur l'image que les anciens ont voulu en donner.

\section{Pouvoir des images, images du pouvoir}

Il faut voir dans le titre de ce chapitre une référence, ou un clin d'œil, à la magistrale synthèse de Zanker. Nulle part n'ont été aussi bien mis en évidence les liens de l'idéologie impériale avec les programmes urbanistiques et iconographiques mis en place sous Auguste à Rome, en Italie et dans les provinces de l'Empire. La visibilité d'Auguste ne s'imposa pas d'entrée de jeu dans l'image urbaine ${ }^{76}$. Elle résulte d'un processus qui transforma l'espace urbain de Rome et des cités de l'Empire au fur et à mesure de la consolidation de son régime et de l'affirmation d'une vocation dynastique précoce. Les travaux de Zanker ont été prolongés à bien des égards par la synthèse de K. Galinsky sur ce qu'il qualifie de « culture augustéenne 77 ». Au contraire de R. Syme, qui parlait de Révolution romaine, il préfere utiliser le vocable d'évolution et suivre au fil du temps le mode de représentation du nouveau régime dans l'art, l'architecture et la littérature d'époque augustéenne. Les multiples changements attestés tout au long de cette période y sont placés sous le signe de l'auctoritas du prince, prestige extraconstitutionnel qui donnait à toute initiative politique d'Auguste une forte valeur morale et à laquelle il est fait directement référence dans les Res Gestae (34.3). Une telle analyse rejoint l'idée de Ferrary selon laquelle la prééminence d'Auguste reposait moins sur une hiérarchie des imperia que sur une auctoritas éclipsant tous les autres organes de l'État romain. La vision de l'historien de l'art et de la littérature rejoint ici celle du spécialiste des institutions politiques. En tant que perpetuus auctor et sous l'apparence trompeuse d'une restauration, Auguste mit en place un nouveau système de valeurs que ses contemporains voulurent imiter ou auxquelles ils voulurent à tout le moins se conformer.

Un des acquis des travaux de Zanker et de Galinsky est d'avoir montré que l'image, en particulier celle qui représente d'une manière ou d'une autre le pouvoir impérial, ne fut pas imposée par et depuis le centre du pouvoir à Rome. Elle apparaît au contraire comme le fruit d'un mode de communication politique entre le nouveau régime et les élites romaines ou provinciales ${ }^{78}$. Il s'agit là d'une avancée scientifique d'autant plus intéressante que, loin d'épuiser toutes les questions, elle a débouché durant cette dernière décennie sur de multiples travaux qui ont prolongé d'une manière

76 Une attention portée aux interventions dans l'urbanisme de Rome durant l'époque triumvirale et les années 20 av. J.-C. a conduit HINARD 2003 à la conclusion que le jeune Octavien/Auguste, loin d'avoir conçu d'emblée un véritable projet urbain, eut à affronter et maîtriser au départ une situation de concurrence créée par le désir des vingt-quatre autres uiri triumphales de cette période d'imprimer à leur tour leur marque sur l'Vrbs. GALINSKY 1996.

78 Cf. entre autres, ZANKER 1991, p. 193-220 et ZANKER 2000, p. 211-245. 
ou d'une autre les résultats auxquels Zanker et Galinsky étaient parvenus. Une mise au point doit être faite d'emblée à propos de l'existence ou non à l'époque augustéenne de ce qu'on a pu appeler la " propagande impériale ». Le choix d'un tel terme, loin d'être innocent, ne manque pas de poser problème pour la période antique, puisqu'il revient à accepter l'idée qu'il existait dès cette époque une action exercée délibérément sur l'opinion publique pour l'amener à adopter le point de vue et les valeurs du pouvoir en place. Zanker et Galinsky ont eux-mêmes évité d'utiliser un tel vocable. Les actes du colloque de Tübingen publié en 2003 par les soins de Gr. Weber et M. Zimmermann ont abouti sur cette question à un résultat identique en soulignant que lorsqu'il est question de l'Antiquité, il vaut mieux renoncer à parler de propagande tant cet outil méthodologique a été usé par les abus de langage et une utilisation trop galvaudée. À un tel concept, jugé anachronique pour l'Antiquité, est préféré le terme, en vogue en Allemagne, d' " auto-représentation " ou celui de "représentation 79 ". En France, Veyne a publié au même moment un article qui allait dans le même sens en montrant dans quelle mesure la formule d' " apparat monarchique " est plus adaptée à l'Empire romain et aux États d'ancien régime que la notion de "propagande ", trop liée à nos sociétés contemporaines ${ }^{80}$. Toutes ces précisions sont parfaitement valides pour des périodes où le pouvoir en place était bien installé. On s'explique dès lors sans peine que celui-ci avait pour vocation à se montrer, sous des formes diverses et pour des raisons multiples, avant de chercher à convaincre. En revanche, pour des périodes de crise comme l'époque augustéenne (ou encore flavienne), les incitations du pouvoir central se firent plus fortes. S’il est vrai que le terme de propagande peut paraître excessif en ce qu'il désigne de nos jours une mise en condition des masses populaires par un régime politique autoritaire, l'idée selon laquelle Auguste (et aussi Tibère au début de son principat) utilisèrent les potentialités de la machine administrative impériale pour faire diffuser à Rome et dans l'Empire la nouvelle idéologie et sa propre image n'est pas non plus sérieusement contestable. Les Tabulae Hebana et Siarensis et le SC de Cn. Pisone patre ont apporté à ce sujet des témoignages éclairants ${ }^{81}$.

L'intérêt porté aux images d'époque augustéenne, que celles-ci aient été imposées par le pouvoir central ou soient le produit d'un dialogue entre Auguste et les élites locales, ne s'est pas démenti ces dernières années. Les résultats d'une telle démarche sont de deux ordres : d'une part, donner la parole aux Italiens et aux provinciaux ${ }^{82}$,

79 Weber \& ZimMERMANN (éd.) 2003, dont l'enquête est centrée sur le Ier siècle de notre ère et où il est souvent question d'Auguste. VEYNE 2002b. Cette étude a été reprise et amplifiée dans VEYNE 2005, p. 379-418. HURLET 2006b.

Cf. STROTHMANN 2000 dont la seconde partie, la plus longue et la plus originale, examine la question de la diffusion à l'échelle de l'Empire de trois notions présentées comme autant de concepts centraux : la restitutio, qui est définie de façon générale comme le rétablissement d'un état antérieur - tant d'un point de vue juridique qu'édilitaire - et renvoie à la volonté bien connue d'Auguste d'apparaître comme le restaurateur de la Res publica; le 
amenés à représenter le pouvoir impérial dans l'espace public (et aussi privé) de leur cité pour des raisons ponctuelles qu'il n'est pas toujours possible de comprendre dans l'état actuel de nos connaissances; d'autre part, souligner le caractère dynastique d'un régime sur la nature duquel les provinciaux ne se sont jamais mépris. La publication de la Tabula Siarensis et du SC de Cn. Pisone patre est venue préciser qu'il fallait chercher la traduction de ce que nous appelons une dynastie dans le terme domus, complété ou non des qualificatifs Augusta ou Diuina, qui présente la particularité d'élargir le cercle familial au-delà de la gens en y incluant les parents par alliance (les adfines) ${ }^{83}$. Les Italiens et les provinciaux s'emparèrent de cette notion centrale pour se représenter le régime augustéen, considérant en fin de compte Auguste et ses " co-régents " moins comme des titulaires de pouvoirs d'essence républicaine - imperium et puissance tribunicienne - que comme des parents d'une famille dont ils avaient accepté la vocation à diriger l'Empire. C'est ce qui ressort en particulier de l'examen attentif accordé ces dernières années aux groupes statuaires, forme de représentation du pouvoir qui associait Auguste aux membres (féminin et masculin) de sa dynastie et dont les témoignages se multiplièrent à l'échelle de l'Empire avec l'ascension politique de Caius César, le fils adoptif du prince, à partir de 6 av. J.-C. ${ }^{84}$. Jules César ne cessa ainsi jamais d'être présent dans l'image publique en raison de la caution divine qu'il garantissait à une dynastie dont il était le fondateur ${ }^{85}$. Les représentations des autres figures - masculines ou féminines - de la domus impériale ont été également étudiées durant cette dernière décennie, depuis Agrippa jusqu'à Germanicus et Drusus le Jeune ${ }^{86}$.

On trouve une des expressions les plus achevées de l'image que le prince voulut donner de son régime dans le programme décoratif du Forum d'Auguste. Dans une

saeculum, qui fait référence au retour de l'âge d'or fêté à Rome en juin 17 av. J.-C. avec la célébration des jeux séculaires et témoigne de l'emprise du pouvoir impérial sur le temps ; le titre de pater patriae, qui fut officiellement décerné à Auguste en tant que surnom le 5 février 2 av. J.-C. et le faisait apparaître comme un nouveau fondateur de Rome.

83 Sur cette question, il faut renvoyer aux travaux de Ph. Moreau et de M. Corbier (cf. en dernier lieu CORBIER 2001).

84 Cf. HuRLET 1997, p. 416-538 qui se limite aux groupes statuaires incluant les " corégents "; Rose 1997 ; BosChung 2002 ; cf. aussi BuXTON \& HANNAH 2005 qui font de l'année 6 ap. J.-C. le point de départ de l'affirmation par le nouveau régime d'une véritable politique dynastique et de la diffusion dans l'Empire de l'image de Caius et Lucius César.

KIENAST 2001 a montré que contrairement à ce qui est parfois affirmé, l'image de César continua à être fortement présente à un titre officiel tout au long de l'époque augustéenne au travers des divers modes de représentation (inscriptions, monnaies, statues, monuments publics, fêtes du calendrier...).

On citera notamment ROMEO 1998 pour ce qui concerne les représentations d'Agrippa (de son vivant et posthumes) ; cf. aussi SCHARF 2001 sur Agrippa Postumus et BARTMAN 1999 sur Livie. 
monographie qui n'a pas volontairement cherché à étudier tous les aspects du problème, M. Spannagel a développé ce qui lui paraît le mieux rendre compte de la signification politique et idéologique de cet ensemble monumental ${ }^{87}$. Ont été choisis trois thèmes majeurs qui y font chacun l'objet d'un long chapitre : les étapes d'aménagement depuis le projet initial jusqu'à l'inauguration officielle en 2 av. J.-C. ; les deux groupes centraux qui représentaient la fuite d'Énée de Troie et Romulus en possession des dépouilles opimes et qui étaient situés au centre des exèdres sur les côtés du temple de Mars Ultor ; la succession de statues localisées le long et au fond des deux portiques latéraux. Ce livre met bien en évidence la complexité du programme iconographique du Forum d'Auguste en se livrant à une analyse sémantique souvent convaincante des innombrables images agencées de manière remarquablement cohérente. L’entreprise de Spannagel a été précédée, et préparée, par une monographie de Zanker (1968), mais elle dépasse en ampleur ce qui a été fait jusqu’alors. Parmi les résultats originaux, il faut en extraire les principaux : datation de la dédicace du temple de Mars Ultor (12 mai au lieu du $1^{\text {er }}$ août) ; lien entre la décision de construire un tel forum et une mesure datée de 17 av. J.-C. qui enjoignit aux triomphateurs d'élever chacun un monument public et fut prise dans le contexte de la célébration des jeux séculaires, la fête du nouveau régime. La monographie de Spannagel a le grand mérite d'insister sur la dimension dynastique du Forum d'Auguste plus que cela n'avait été fait jusqu'alors. Il a été maintes fois répété que le programme décoratif visait à présenter la prise du pouvoir par Auguste et le régime fondé par ce dernier comme l'aboutissement d'une histoire romaine déjà longue de plusieurs siècles. Il apparaît désormais que le message politique délivré par cet ensemble monumental était également tourné vers l'avenir. Il s'agissait de veiller à la continuité de la dynastie et de donner aux principes à venir des modèles - exemplum selon la version des Res Gestae, exemplar d'après le texte d'un édit reproduit par Suétone, Aug., 31.5 - fondés sur les actions d'Auguste, de ses nombreux ascendants et des grandes figures de l'histoire romaine.

Un autre ensemble architectural emblématique de l'idéologie augustéenne est l'ara Pacis Augustae, l'autel de la Paix Auguste, dont la construction fut décidée le 4 juillet 13 et qui fut dédicacé le 30 janvier 9 av. J.-C. L'analyse de ce monument a été renouvelée par les fines analyses de G. Sauron, développées en particulier dans une monographie récente dont le titre est tout un programme : L'histoire végétalisée ${ }^{88}$. Les rinceaux situés dans la partie inférieure des quatre faces de l'enceinte en marbre y font l'objet d'une étude détaillée qui cesse d'en faire de purs ornements pour décrypter leur sémantique historique et leur signification dynastique. L'acanthe est élevée au rang d'emblème du nouveau pouvoir et triomphe partout de la vigne et du lierre, les végétaux de Dionysos assimilés à Antoine. Sauron scrute les détails avec une telle attention 
qu'il identifie au détour des volutes des événements tels que la fuite de la flotte de Cléopâtre et d'Antoine à Actium derrière les touffes retournées de feuilles de lierre, le double suicide des vaincus par le biais de la représentation des vrilles-aspics et de leur tombeau végétalisé ou encore les naissances de Caius et de Lucius César à travers la présence d'un ove d'où retombent deux jeunes pousses. Le message de fond tient dans l'affirmation du lien entre la prise du pouvoir par Auguste et le retour d'un âge d'or, matérialisé par la cérémonie des jeux séculaires de 17 av. J.-C. et incarné par des motifs végétaux qui font apparaître une nature apaisée comme reflet d'un nouvel ordre stable et hiérarchisé. Cette nouvelle esthétique du décor, qui définit un nouveau classicisme, contraste avec les compositions tourmentées du dernier siècle de la République romaine qui donnent l'image d'un monde entièrement soumis au chaos. Derrière une telle évolution de l'art ornemental est reconnue l'influence du prince et de plusieurs de ses proches, en l'occurrence les poètes Virgile et Horace.

À côté de l'étude de l'image matérielle, il y a place pour une meilleure appréhension de la représentation mentale que les contemporains se faisaient du nouveau régime. Les sources littéraires d'époque augustéenne continuent à faire l'objet de la plus grande attention et il faut signaler à cet égard la parution d'une étude de B. Mineo sur les liens entre la rédaction de l'histoire romaine de Tite-Live et le contexte dans lequel cette œuvre fut conçue ${ }^{89}$. Il y est en particulier démontré comment le présent augustéen influa sur l'écriture du passé guidée par une vision analogique qui faisait des grandes figures de l'histoire de Rome (Énée, Évandre, Romulus, Camille ...) autant de préfigurations d'Auguste. Au nombre des apports de cette enquête, le découpage de l'Ab Vrbe condita en deux cycles complets de 365 années (les Grandes Années) éclaire le fonctionnement d'une palingénésie livienne qui fait de l'instauration du principat le point de convergence idéologique de l'œuvre.

\section{Prospectives}

Le dernier mot est loin d'avoir été dit à propos d'Auguste. S'il est acquis que l'approche juridique ne peut rendre compte de tous les aspects du principat tel qu'il fut conçu par son fondateur, il ne faudrait pas pour autant renoncer à l'idée fondamentale selon laquelle l'exercice du pouvoir ne peut se concevoir en dehors de tout cadre institutionnel ${ }^{90}$. C'est cette voie qui m'est apparue à l'examen à la fois comme la plus sûre, la plus

Mineo 2006.

90 Cf. à ce sujet les propos de P. Grenade, publiés en 1961 et toujours d'actualité : «Il y a donc deux exagérations à éviter et les modernes doivent, adoptant l'attitude de Pascal devant la raison, se défier à propos des problèmes constitutionnels des deux excès contraires : exclure le droit public, n'admettre que le droit public » (p. IX). 
équilibrée et la seule à être réellement novatrice ${ }^{91}$. L'analyse de la place du consensus dans le régime augustéen le montre bien. Si l'insistance récente sur un tel concept est justifiée eu égard au contenu de sources récemment publiées, elle ne doit s'exercer ni aux dépens d'autres fondements idéologiques ni au détriment d'une analyse des formes légales par lesquelles se manifestait l'unanimité à l'égard d'Auguste. L'ouvrage de A. Pabst est à ce jour le seul à avoir (ré)concilié deux aspects du pouvoir impérial qu'elle appelle "Principatskomponente" et "Imperiumskomponente» et qui peuvent être alternativement pris en compte selon que l'on parle des fondements sociologiques du principat ou de sa mise en forme juridique ${ }^{92}$. Il faut maintenant aller au-delà de ce constat et étudier l'articulation entre deux composantes qui ne sont pas antinomiques. Il faut admettre que la tâche du spécialiste d'Auguste est devenue plus compliquée. S'il ne peut se permettre de négliger l'étude des fondements institutionnels du pouvoir d'Auguste, il doit dans le même temps tenir compte des travaux récents des tenants de la sociologie historique. La pluridisciplinarité des études augustéennes ne doit pas s’arrêter là. Le renouvellement est venu et viendra des recherches archéologiques, à la condition d'être sensible au contexte politique et idéologique dans lequel les vestiges matériels mis au jour s'inscrivaient. La numismatique reste également un chantier largement ouvert notamment depuis la publication à partir de 1992 du premier tome du monnayage provincial d'époque impériale dont les potentialités ont été à peine exploitées par les historiens ${ }^{93}$. Il faut enfin

91 Cf. les propos de De MARTINO 1998, p. 140-141 : « Mi sia permesso di ricordare che ad esse [= le condizioni materiali della società del tempo] chi scrive ha tentato di collegare anche gli sviluppi costituzionali, senza considerarli come i soli fattori determinanti, come talvoltà ingiustamente mi è stato attribuito, ma necessari per venir fuori dal formalismo delle definizioni astratte e logiche e porre le istituzioni, che sone create dagli uomini e da essi governate, nella realtà dei rapporti. Nel ricostruire pazientamente l'esercizio del potere nei vari campi e quindi risalire alle espressioni giuridiche formali o non che siano si puó definire l'ordinamento nel suo valore concreto di costituzione sostanziale. Questo metodo è il solo realmente innovativo. "

92 PABST 1997.

93 Cf. Roman Provincial Coinage, t. I, éd. par A. Burnett, M. Amandry et P.P. Ripolles, Londres-Paris. Il est certain qu'un réexamen attentif du monnayage impérial apportera également du neuf (on se reportera au volume I du Roman Imperial Coinage, éd. par C.H.V. Sutherland, Londres, 19842). Parmi les études récentes à signaler et sans prétendre à l'exhaustivité, on citera la publication des monnaies de Teutobourg avec un point sur la circulation augustéenne par BERGER 1996, le volume Trésors Monétaires XX (trésors de Meussia et d'Angers) qui est important en particulier pour le monnayage à l'effigie des Césars et la réédition du catalogue du fonds BNF de monnaies augustéennes par J.-B. Giard avec une nouvelle et utile préface (Monnaies de l'Empire romain. I Auguste. Catalogue, Paris, 20013, auquel il faut y joindre le CR de Burnett dans RN 2003 qui souligne ce qu'il reste à faire), l'étude de LEVICK 1999 et la synthèse complète de WOLTERS 2003 sur des questions aussi centrales que l'identification de l'autorité responsable du choix des différents types sous le Haut-Empire (prince ou tresuiri monetales ?) ou encore le degré de lisibilité des images et des légendes par ceux qui, en grand nombre, manipulaient les monnaies. 
renouer la collaboration avec les philologues de manière à envisager une étude des auteurs augustéens, en particulier des poètes, dans une perspective historique. R. Syme a ouvert cette voie en consacrant notamment une monographie à l'histoire chez Ovide (History in Ovid, Oxford, 1978) ; B. Mineo a prolongé ce type d'enquête en replaçant la rédaction de la somme monumentale de Tite-Live dans son environnement politique. Nul doute qu'il y aura également beaucoup d'enseignements à tirer d'une relecture orientée des œuvres de Virgile, Properce, Tibulle ou encore Horace 94.

\author{
Frédéric HURLET \\ Université de Nantes \\ UFR d'Histoire, Histoire de l'Art et Archéologie \\ EREMAM (Équipe de recherche sur les Échanges dans la \\ Méditerranée antique et médiévale) \\ Chemin de la Censive du Tertre \\ $B P 81227$ \\ F-44312 Nantes Cedex 3 \\ Frederic.Hurlet@univ-nantes.fr
}

\title{
Bibliographie
}

ADCOCK, F.E. (1951) : “The Interpretation of Res Gestae Diui Augusti, 34.1”, CQ, 45, p. 130 135.

ALFÖLDY, G. (1991) : "Augustus und die Inschriften : Tradition und Innovation. Die Geburt der imperialen Epigraphik", Gymnasium, 98, p. 289-324 [traduction italienne dans Scienze dell'antichità, 5, 1991, p. 573-600].

- (2000) : "Das neue Edikt des Augustus aus El Bierzo in Hispanien", ZPE, 131, p. $177-205$.

Bartman, E. (1999) : Portraits of Livia. Imaging the Imperial Woman in Augustan Rome, Cambridge.

BeHne, Fr. (1999) : Heinrich Siber und das römische Staatsrecht von Theodor Mommsen. Ein Beitrag zur Rezeptionsgeschichte Mommsens im 20. Jahrhundert, Hildesheim - Zurich - New York.

Berger, F. (1996) : Kalkriese 1. Die römischen Fundmünzen, Mayence.

BleICKen, J. (1990) : Zwischen Republik und Prinzipat. Zum Charakter des zweiten Triumvirats, Göttingen.

- (1998) : Augustus. Eine Biographie, Berlin.

Boschung, D. (2002) : Gens Augusta. Untersuchungen zur Aufstellung, Wirkung und Bedeutung der Statuengruppen des julisch-claudischen Kaiserhauses, Mayence.

94 On trouvera, au sujet des poètes, un premier traitement utile dans le chapitre $\mathrm{V}$ de GALINSKY 1996, p. 225-287, consacré en grande partie aux poètes d'époque augustéenne. 
BRAndT, H. (2000) : “Octavian/Augustus - Totengräber und Friedensfürst”, dans Von Romulus zu Augustus. Große Gestalten der römischen Republik, éd. par K.-J. Hölkeskamp et E. SteinHölkeskamp, Munich, p. 365-376.

Buxton, (Br.) \& Hannah, R. (2005) : "OGIS 458, the Augustan Calender and the Succession”, dans Studies in Roman Literature and Roman History, XII, éd. par C. Deroux, Bruxelles, p. 290-306.

Castritius, H. (1982) : Der römische Prinzipat als Republik, Husum.

Chastagnol, A. (1992) : Le Sénat romain à l'époque impériale, Paris.

Cogitore, I. (2002) : La légitimité dynastique d'Auguste à Néron à l'épreuve des conspirations, Rome.

Corbier, M. (2001) : "Maiestas domus Augustae", dans Varia Epigraphica. Atti del Colloquio Internazionale di Epigrafia, Bertinoro, 8-10 giugno 2000, éd. par G. Angeli Bertinelli et A. Donati, Faenza, p. 155-199.

Cosme, P. (2005) : Auguste, Paris.

Costabile, F. \& Licandro, O. (2000) : “Tessera Paemeiobrigensis. Un nuovo editto di Augusto dalla Transduriana prouincia e l'imperium proconsulare del princeps”, Minima Epigraphica et papyrologica, 3, 2, p. 147-235.

Cotton, H. M. \& Yakobson, A. (2002) : "Arcanum imperii: The Powers of Augustus", dans Philosophy and Power in the Graeco-Roman World. Essays in Honour of M. Griffin, éd. par G. Clark et T. Rajak, Oxford, p. 193-209.

CraWford, M.H. éd. (1996) : Roman Statutes, Londres.

Cresci Marrone, G. éd. (1998) : Temi augustei, Atti dell'incontro di studio, Venezia, 5 giugno 1996, Amsterdam.

Crook, J.A. (1996) : "Political History, 30 B.C. to A.D. 14", dans CAH, $\mathrm{X}^{2}$, Cambridge.

De MARTINO, Fr. (1998) : "Considerazioni su alcuni temi di storia costituzionale romana", dans Hommage à la mémoire de A. Magdelain, éd. par M. Humbert et Y. Thomas, Paris, p. 133149.

Demougin, S. (1988) : Lordre équestre sous les Julio-Claudiens, Rome.

Dettenhofer, M. H. (2000) : Herrschaft und Widerstand im augusteischen Principat. Die Konkurrenz zwischen res publica und domus Augusta, Stuttgart.

DreW-Bear, Th. \& ScheID, J. (2005) : "La copie des Res Gestae d'Antioche de Pisidie", ZPE, 154, p. 217-260.

ECK, W. (1998) : Augustus und seine Zeit, Munich [4e éd. en 2006 ; il existe une traduction italienne publiée en 2000, une traduction en castillan datée de 2001 et une traduction anglaise parue en 2004].

Eck, W., Caballos, A. et Fernández, F. (1996) : Das S.C. de Cn. Pisone patre, Munich [il existe une version espagnole moins développée : El senadoconsulto de Gneo Pisón padre, Séville, 1996].

Ferrary, J.-L. (2001a) : “À propos des pouvoirs d'Auguste”, CCGG, 12, p. 101-154.

- (2001b) : "Les pouvoirs d'Auguste : l'affranchissement de la limite du pomerium", dans Rome, les Césars et la Ville aux deux premiers siècles de notre ère. Études d'histoire politique, sociale et religieuse, éd. par N. Belayche, Rennes, p. 9-22.

- (2003) : "Res publica restituta et les pouvoirs d'Auguste", in Fondements et crises du pouvoir, éd. par S. Franchet d'Esperey - V. Fromentin - S. Gotteland - J.-M. Roddaz, Bordeaux, p. $419-428$. 
FlaIG, E. (1992) : Den Kaiser herausfordern. Die Usurpation im römischen Reich, Francfort-New York.

Fraschetti, A., (1998) : Augusto, Bari.

— éd. (2000) : La commemorazione di Germanico nella documentazione epigrafica. Convegno Internazionale di Studi (Cassino, 1991), Rome.

GALINSKY, K. (1996) : Augustan Culture : an Interpretive Introduction, Princeton.

Giovannini, A. (1983) : Consulare imperium, Bâle.

- (1999) : "Les pouvoirs d'Auguste de 27 à 23 av. J.-C. Une relecture de l'ordonnance de Kymè de l'an 27 (IK 5, n 17)", ZPE, 124, p. 95-102.

- (éd.) (2000) : La révolution romaine après Ronald Syme : bilans et perspectives, VandouvresGenève.

Girardet, Kl. M. (1990) : "Die Entmachtung des Konsulates im Übergang von der Republik zur Monarchie und die Rechtsgrundlagen des augusteischen Prinzipats", dans Pratum Saraviense, Festschrift für P. Steinmetz, éd. par W. Görler et S. Koster, Stuttgart, p. 89-126 [= Girardet 2007, p. 385-423].

— (1992) : "Zur Diskussion um das imperium consulare militiae im 1. Jh. v. Chr.", CCGG, 3, p. 213-220 [= Girardet 2007, p. 425-433].

- (2000a) : "Imperium " maius" : politische und verfassungsrechtliche Aspekte. Versuch einer Klärung”, dans Giovannini éd. 2000, p. 167-236 [= Girardet 2007, p. 461-521].

- (2000b) : "Das Edikt des Imperator Caesar Augustus in Suetons Augustusvita 28,2. Politisches Programm und Publikationszeit", ZPE, 131, p. 231-243 [= Girardet 2007, p. 363-384].

- (2007) : Rom auf demWeg von der Republik zum Prinzipat, Bonn.

GRENADE, P. (1961) : Essai sur les origines du principat, Paris.

Gros, P. (1996) : L'architecture romaine, t. I, Paris.

- (2001) : "Nunc tua cinguntur limina: l'apparence de l'accueil et la réalité du filtrage à l'entrée des Forums impériaux de Rome”, dans Rome et ses provinces. Genèse et diffusion d'une image du pouvoir. Hommages à J.-Ch. Balty, éd. par C. Evers et A. Tsingarida, Bruxelles, p. 129-140.

— (2005) : "La ville comme symbole. Le modèle central et ses limites", dans Histoire de la civilisation romaine, éd. par H. Inglebert, Paris, p. 155-232.

HinARD, Fr. (2003) : "Entre République et principat. Pouvoir et urbanité", dans Laurea internationalis. Festschrift für Jochen Bleicken zum 75. Geburtstag, éd. par Th. Hantos, Stuttgart, p. 331-358.

HÖLSCHER, T. (2000) : “Augustus und die Macht der Archäologie”, dans Giovannini éd. 2000, p. 237-273 (et la discussion p. 274-281).

HURLET, Fr. (1997) : Les collègues du prince sous Auguste et Tibère. De la légalité républicaine à la légitimité dynastique, Rome.

— (2000) : "Les sénateurs dans l'entourage d'Auguste et de Tibère. Un complément à plusieurs synthèses récentes sur la cour impériale", dans $R P h, 74$, p. 123-150.

- (2001) : "Les auspices d'Octavien/Auguste", CCGG, 12, p. 155-180.

- (2002) : "Le consensus et la concordia en Occident (Irr-III" siècles ap. J.-C.). Réflexions sur la diffusion de l'idéologie impériale", dans Idéologies et valeurs civiques dans le monde romain. Hommage à Claude Lepelley, éd. par H. Inglebert, Paris, p. 163-178.

- (2006a) : Le proconsul et le prince d'Auguste à Dioclétien, Bordeaux. 
- (2006b) : "Les modalités de la diffusion et de la réception de l'image et de l'idéologie impériale sous le Haut-Empire en Occident", dans La transmission de l'idéologie impériale dans l'Occident romain [128 Congrès des sociétés historiques et scientifiques (Relations, échanges et coopération en Méditerranée), Bastia, avril 2003], éd. par M. Navarro Caballero et J.-M. Roddaz, Bordeaux-Paris, p. 49-68.

- (2006c) : "Auguste et Pompée", Athenaeum, 94, p. 467-485.

InSTINSKY, H. U. (1940) : "Consensus uniuersorum", Hermes, 75, p. 265-278 [= H. Oppermann (éd.), Römische Wertbegriffe, Darmstadt, 1967, p. 209-228].

ITGENSHORST, T. (2004) : "Augustus und der republikanische Triumph. Triumphalfasten und summi uiri-Galerie als Instrumente der imperialen Machtsicherung”, Hermes, 132, p. 436458.

JACQUeS, Fr. \& SCHeID, J. (1990) : Rome et l'intégration de l'Empire, 44 av. J.-C. - 260 ap. J.-C., t. I (Les structures de l'empire romain), Paris.

Kienast, D. (19993) : Augustus. Prinzeps und Monarch, Darmstadt (la première édition date de 1982).

- (2001) : "Augustus und Caesar", Chiron, 31, p. 1-26

Kromayer, J. (1888) : Die rechtliche Begründung des Principats, Marburg.

LACEY, W.K. (1996) : Augustus and the Principate. The Evolution of the System, Leeds.

LE GALL, J. (1985) : "Le serment à l'empereur : une base méconnue de la tyrannie impériale sous le Haut-Empire", Latomus, 44, p. 767-783 [reproduit dans CCGG, 1, 1990, p. 165180].

Le Roux, P. (2001) : “L'edictum de Paemeiobrigensibus. Un document fabriqué ?", Minima epigraphica et papyrologica, 6, p. 331-363.

LevicK, B. (1999) : "Messages on the Roman Coinage : Types and Inscriptions", dans Roman Coins and Public Life under the Empire, éd. par G.M. Paul \& M. Ierardi, Ann Arbor, p. 4160.

Linderski, J. (1990) : "Mommsen and Syme : Law and Power in the Principate of Augustus", dans Between Republik and Empire. Interpretations of Augustus and his Principate, éd. par K. A. Raaflaub and M. Toher, Berkeley-Los Angeles-Oxford, p. 51-52 [= Roman Questions. Selected Papers, Stuttgart, 1995, p. 32-43].

Millar, F. (2000) : “The First Revolution : Imperator Caesar, 36-28 BC”, dans Giovannini éd. 2000, p. 1-38.

- (2002) : Rome, the Greek World, and the East, Vol. I. The Roman Republic and the Augustan Revolution, éd. par H.M. Cotton et G.M. Rogers, Chapell-Hill - Londres.

Mineo, B. (2006) : Tite-Live et l'histoire de Rome, Paris.

MommSen, Th. DPR : Le droit public romain, trad. fr., Paris, De Boccard, 1984 (Römisches Staatsrecht, 1re éd., Leipzig, 1871 et 1875 pour les t. I-II, 1887-1888 pour le t. III ; 3e éd., Leipzig, 1887, pour les t. I-II).

NiCOLET, Cl. (1976) : "Le cens sénatorial sous la République et sous Auguste”, JRS, 66, p. 2138 [republié dans Des ordres à Rome, Paris, 1985, p. 143-174].

- (1988) : L'inventaire du monde. Géographie et politique aux origines de l'Empire romain, Paris.

— (1992) : "Autour de l'imperium", CCGG, 3, p. 163-166.

PABST, A. (1997) : Comitia Imperii. Ideelle Grundlagen des römischen Kaisertums, Darmstadt.

PANI, M. (2001) : "L'imperium de Tiberio principe", dans Epigrafia e territorio. Politica e società. Temi di antichità romane, VI, éd. par M. Pani, Bari, p. 253-262. 
PASCHOud, Fr. (2005) : “À propos d'imperium maius: nil sub sole nouum”, ZPE, 153, p. 280282.

Pelham, H.F. (1888) : “The 'imperium' of Augustus and his Successors”, Journal of Philology, 17, p. 27-52 [= Essays on Roman History, Oxford, 1911, p. 60-88].

RaINer, J.M. (2006) : Römisches Staatsrecht. Republik und Prinzipat, Darmstadt.

RenucCI, P. (2003) : Auguste le révolutionnaire, Paris.

Rich, J. W. \& Williams, J. H. C. (1999) : "Leges et iura P. R. Restituit : a New Aureus of Octavian and the Settlement of 28-27 BC", NC, p. 169-213.

RichardsOn, J.S. (2002) : "The New Augustan Edict from Northwest Spain”, JRA, 15, p. 411415.

RODDAZ, J.-M. (1992) : “Imperium : nature et compétences à la fin de la République et au début de l'Empire", $C C G G$, 3, p. 189-211

- (2003) : "La métamorphose : d'Octavien à Auguste", in Fondements et crises du pouvoir, éd. par S. Franchet d'Esperey - V. Fromentin - S. Gotteland - J.-M. Roddaz, Bordeaux, p. 397418.

Rohr VIO, Fr. (2000) : Le voci del dissenso. Ottaviano Augusto e i suoi oppositori, Padoue.

Romeo, I. (1998) : Ingenuus leo : l'immagine di Agrippa, Rome.

Rose, Ch. Br. (1997) : Dynastic Commemoration and Imperial Portraiture in the Julio-claudian Period, Cambridge.

Rowe, Gr. (2002) : Princes and Political Cultures. The New Tiberian Senatorial Decrees, Ann Arbor.

RÜPKE, J. (1995) : Kalender und Öffentlichkeit : Die Geschichte der Repräsentation und religiösen Qualifikation von Zeit in Rom, Berlin.

— (2003) : "L'histoire des fasti romains : aspects médiatiques et politiques", RHD, 81, p. 125139.

- (2005) : Fasti sacerdotum. Die Mitglieder der Priesterschaften und das sakrale Funktionspersonal römischer, griechischer, orientalischer und jüdisch-christlicher Kulte in der Stadt Rom von 300 v. Chr. bis 499 n. Chr., Stuttgart.

SÁnchez-Ostiz, A. (1999) : Tabula Siarensis. Edición, traducción y comentario, Pampelune.

SAuron, G. (2000) : L’histoire végétalisée. Ornement et politique à Rome, Paris.

- (2004) : "Actualité du Forum d'Auguste", REL, 82, p. 25-31.

Scharf, R. (2001) : Agrippa Postumus. Splitter einer historischen Figur, Landau.

SCHEID, J. (1999) : "Auguste et le grand pontificat. Politique et droit sacré au début du principat", $R H D, 77$, p. $1-19$.

— (2003) : "Les vœux pour le salut d'Octavien de 32 av. J.-C. (RGDA 9,1)", dans Laurea internationalis. Festschrift für Jochen Bleicken zum 75. Geburtstag, éd. par Th. Hantos, Stuttgart, p. 359-365

- (2005) : Quand faire, c'est croire. Les rites sacrificiels des Romains, Paris.

SCHNEGG-KÖHLER, B. (2002) : "Die augusteischen Säkularspiele", Archiv für Religionsgeschichte, 4.

SOUTHERN, P. (1998) : Augustus, Londres.

SpANNAGEL, M. (1999) : Exemplaria principis. Untersuchungen zu Entstehung und Ausstattung des Augustusforums, Heidelberg. 
SpÄTH, Th. (1999) : "Nouvelle histoire ancienne ? Sciences sociales et histoire romaine : à propos de quatre récentes publications allemandes (note critique)", Annales HSS, p. $1137-$ 1156.

Strothmann, M. (2000) : Augustus - Vater der res publica. Zur Funktion der drei Begriffe restitutio - saeculum - pater patriae im augusteischen Prinzipat, Stuttgart.

Stylow, A.U. \& Corzo Pérez, S. (1999) : "Eine neue Kopie des Senatus consultum de Cn. Pisone patre", Chiron 29, p. 23-28.

Suspène, A. (2001) : “Tiberius Claudianus contre Agrippa Postumus : autour de la dédicace du temple des Dioscures", $R P h, 75$, p. 99-124.

Swan, P.M. (2004) : The Augustan Succession : an Historical Commentary on Cassius Dio's Roman History, Books 55-56 (9 B.C. - A.D. 14), New York.

TARPIN, M. (2003) : "M. Licinius Crassus imperator, et les dépouilles opimes de la République", $R P h, 77$, p. $275-311$.

VeYne, P. (2002a) : "L'empereur, ses concitoyens et ses sujets", dans Idéologies et valeurs civiques dans le monde romain. Hommage à Claude Lepelley, éd. par H. Inglebert, Paris, p. 49-64.

— (2002b) : "Lisibilité des images, propagande et apparat monarchique dans l'Empire romain", RH, p. 3-30.

- (2005) : L'Empire gréco-romain, Paris.

Wallace-Hadrill, A. (1996) : "The Imperial Court", dans $C A H \mathrm{X}^{2}$, Cambridge, p. 283-308.

Weber, (Gr.) \& Zimmermann, (M.) éd. (2003) : Propaganda - Selbstdarstellung - Repräsentation im römischen Kaiserreich des I. Jhs. n. Chr., Stuttgart.

Winterling, A. (1999) : Aula Caesaris. Studien zur Institutionalisierung des römischen Kaiserhofes in der Zeit von Augustus bis Commodus (31 v. Chr. 192 n. Chr.), Munich.

Wolters, R. (2003), "Die Geschwindigkeit der Zeit und die Gefahr der Bilder : Münzbilder und Münzpropaganda in der römischen Kaiserzeit”, dans Weber \& Zimmermann 2003, p. $175-204$.

ZANKer, P. (1968) : Forum Augustum. Das Bildprogramm, Tübingen [reproduite dans Saeculum Augustum, III, éd. par G. Binder, Darmstadt, 1991, p. 60-111].

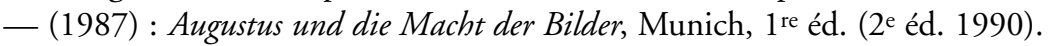

- (1991) : "Immagini e valori collettivi", dans Storia di Roma, II, 2, éd. par A. Schiavone, Turin, p. 193-220.

- (2000) : "Il mondo delle immagini e la comunicazione", dans Storia di Roma dall'antichità ad oggi. Roma antica, éd. par A. Giardina, Bari, p. 211-245 [=Un'arte per l'impero. Funzione e intenzione delle immagini nel mondo romano, Milan, 2002, p. 9-37].

ZEHNACKER, H. (2003) : "Quelques remarques sur le revers du nouvel aureus d'Octavien (28 av. J.-C.)”, BSFN, 58-1, p. 1-3. 